\title{
Twisted flux tube emergence from the convection zone to the corona
}

\author{
Juan Martínez-Sykora \\ and \\ Viggo Hansteen \\ and \\ Mats Carlsson \\ Institute of Theoretical Astrophysics, University of Oslo, P.O. Box 1029 Blindern, N-0315 \\ Oslo, Norway
}

\begin{abstract}
Three dimensional numerical simulations of the emergence of a horizontal magnetic flux tube with different twist levels are carried out in a computational domain spanning the upper layers of the convection zone to the lower corona. We use the Oslo Staggered Code (OSC) to solve the full MHD equations with non-grey and non-LTE radiative transfer and thermal conduction along the magnetic field lines. The emergence of the magnetic flux tube input at the bottom boundary into a weakly magnetized atmosphere is presented. The photospheric and chromospheric response is described with magnetograms, synthetic continuum images at different wavelengths, as well as synthetic Ca II H-line images, and velocity field distributions.

The emergence of a magnetic flux tube into such an atmosphere results in varied atmospheric responses. In the photosphere the granular size increases when the flux tube approaches from below, as has been reported previously in the literature. In the convective overshoot region some $200 \mathrm{~km}$ above the photosphere adiabatic expansion produces cooling, darker regions with the structure of granulation cells. We also find collapsed granulation in the boundaries of the rising flux tube. Once the flux tube has crossed the photosphere, bright points related with concentrated magnetic field, vorticity, high vertical velocities and heating by compressed material are found at heights up to $500 \mathrm{~km}$ above the photosphere. At greater heights in the magnetized chromosphere, we find that the rising flux tube produces a large, cool, dim, magnetized bubble that tends
\end{abstract}


to expel the usual chromospheric oscillations. In addition the rising flux tube dramatically increases the chromospheric scale height, pushing the transition region and corona aside such that the chromosphere extends up to $6 \mathrm{Mm}$ above the photosphere in the center of the rising flux tube. The emergence of magnetic flux tubes through the photosphere and subsequent rise through the chromosphere up to the bottom of the lower corona is a relatively slow process, taking of order 1 hour to complete. In later papers we intend to discuss the merging of the newly emerged field with the pre-existing coronal field and its diagnostic consequences.

Subject headings: Magnetohydrodynamics MHD - Methods: numerical — Radiative transfer — Sun: atmosphere — Sun: magnetic field

\section{Introduction}

One of the stated goals of the newly launched Hinode satellite is to follow the evolution of magnetic flux from the moment of emergence through the photosphere and into the chromosphere and corona. Such observations come at a timely moment as they should prove a fertile testing ground for the 3D numerical models of flux emergence that are now becoming available due to the technological development of massively parallel computers and algorithms to utilize these.

A great variety of simplified simulations have been developed in order to study the different phases of flux emergence. Originally, early simulations were performed in $1 \mathrm{D}$ due to computational limitations. Studies of rising flux tubes were carried out to gain an understanding of the emergence latitudes of active regions, their tilt angles as well as asymmetry between leading and following polarities and magnetic forces such as tension (Moreno-Insertis 1986; Fan et al. 1993; Caligari et al. 1995). The early 80's saw the appearance of $2 \mathrm{D}$ simulations where Schüssler (1979) and Longcope et al. (1996) found that an emerging magnetic

flux tube without twist is fragmented. Moreno-Insertis \& Emonet (1996) investigated the effect of the surrounding flows on the flux tube's rise to the surface and demonstrated that a minimum twist in the tube was required in order to suppress the conversion of the tube into vortex pairs (Lites et al. 1995). Emonet et al. (2001) studied the Von Kármán vortex street generated in the boundary layer of the tube (Dorch \& Nordlund 1998). In the 90's 2D simulations were extended to 3D. Fan et al. (1998) investigated flux tubes moving through an adiabatic stratification found that the degree of twist must be large enough to avoid fragmentation yet small enough to avoid the kink instability. There are also other 3D emergence models that while not including radiative transfer, nor any detailed treatment of convection cells below the photosphere, have added schematic descriptions of the chromosphere 
and corona (Matsumoto et al. 1998; Fan 2001; Magara 2004, 2006; Manchester et al. 2004; Archontis et al. 2004) in order to gain a handle on the effects to be expected.

On the other hand, in the last decade more realistic 3D MHD models of active region flux emergence have been developed up to the photosphere. Cheung et al. (2007) consider flux emergence through a convective layer including detailed photospheric radiative transfer, but excluding the layers above the photosphere. Other simulations involve solar surface magnetoconvection (Stein \& Nordlund 2002, 2006; Stein et al. 2003; Vögler et al. 2005), but do not consider flux emergence from below.

The description of the outer layers of the solar atmosphere are also growing in sophistication. Gudiksen \& Nordlund (2005) recently performed realistic simulations of the solar corona driven by a stochastic approximation of granulation. Hansteen (2004); Hansteen et al. (2007) and Abbett (2007) studied 3D MHD simulations with a convective layer below the photosphere and included upper atmosphere (chromosphere, transition region and lowercorona) with the radiative losses in the quiet Sun and conduction along the magnetic field treated in detail.

In this paper our goal is to study horizontal flux tube emergence from the upperconvection zone to the lower corona in a realistic 3D MHD model. The model is designed to have a corona driven by magnetoconvection. The radiative losses from the photosphere and lower chromosphere are computed in a non-grey manner and scattering in the chromosphere is handled to a certain approximation (Nordlund 1982; Skartlien 2000). For the upper-chromosphere and corona we assume effectively thin radiative losses. A flux tube is injected into the bottom boundary of an atmosphere that contains a preexisting but weak magnetic field. We follow the evolution of the flux tube as it rises through the various atmospheric layers: How much flux enters the differing regions? When do we see observational signatures of flux emergence? Where and how does the reconnection process happen? How does this affect chromospheric and coronal dynamics and structure? are questions we will discuss.

In section 2 we summarize how the MHD equations with radiative transfer, thermal conduction, viscosity and resistivity are solved by the Oslo Staggered Code. Section 3 shows the initial and boundary conditions of our numerical box for the different simulations. We describe in more detail how the magnetic flux tube is input in the bottom boundary. The results of our simulations are described in section 4 centered on the different events observed in the different layers of the simulation box. We start with the photosphere, then treat the reverse granulation layer, the end of the overshooting and beginning of the chromosphere and finally the upper chromosphere/transition region and lower corona. 


\section{Equations and Numerical Method}

In order to model the rise of magnetic flux tubes through the upper convection layer and their emergence through the photosphere and into the chromosphere and corona we solve the equations of MHD using the Oslo Stagger Code (OSC):

$$
\begin{gathered}
\frac{\partial \rho}{\partial t}+\nabla(\rho \mathbf{u})=0 \\
\frac{\partial \mathbf{u}}{\partial t}+(\mathbf{u} \nabla) \mathbf{u}+\frac{1}{\rho} \nabla(P+\underline{\tau})=+\frac{\mathbf{J} \times \mathbf{B}}{\rho}+\mathbf{g} \\
\frac{\partial(e)}{\partial t}+\nabla(e \mathbf{u})+\mathbf{u} \nabla P=\nabla \mathbf{F}_{r}+\nabla \mathbf{F}_{c}+Q_{\text {Joule }}+Q_{v i s c} \\
\frac{\partial \mathbf{B}}{\partial t}=\nabla \times(\mathbf{u} \times \mathbf{B})-\nabla \times(\underline{\underline{\eta} \mathbf{J}})
\end{gathered}
$$

where $\rho$ represents the mass density, $\mathbf{u}$ the fluid velocity, $P$ the gas pressure, $\mathbf{J}$ the current density, $\mathbf{B}$ the magnetic field, $\mathbf{g}$ gravitational acceleration, and $e$ the internal energy. The viscous stress tensor is written $\underline{\underline{\tau}}$ and the resistivity $\underline{\underline{\eta}} . \mathbf{F}_{r}$ represents the radiative flux, $\mathbf{F}_{c}$ represents the conductive flux, and $Q_{\text {Joule }}$ and $Q_{\text {visc }}$ are the joule heating and viscous heating respectively.

These equations are solved using an extended version of the numerical code described in Dorch \& Nordlund (1998); Mackay \& Galsgaard (2001) and in more detail by Nordlund \& Galsgaard at http://www.astro.ku.dk/ kg and Hansteen et al. (2007). Extended in this case means the inclusion of thermal conduction along the magnetic field, non-grey, non-LTE radiative transfer and characteristic boundary conditions on the upper and lower boundaries. The latter allow the passage of waves out of the computational box while at the same time allowing the specification of an input magnetic field and entropy at the lower boundary.

In short, the code functions as follows: The variables are represented on staggered meshes, such that the density and the internal energy are volume centered, the magnetic field components and the momentum densities $\mathbf{p}=\rho \mathbf{u}$ are face centered, while the electric field $\mathbf{E}$ and the current density are edge centered. A sixth order accurate method involving the three nearest neighbor points on each side is used for determining the partial spatial derivatives. In the cases where variables are needed at positions other than their defined positions a fifth order interpolation scheme is used. The equations are stepped forward in time using the explicit 3rd order predictor-corrector procedure defined by Hyman et al. (1979), modified for variable time steps. In order to suppress numerical noise, high-order artificial diffusion is added both in the forms of a viscosity and in the form of a magnetic diffusivity. 
The radiative flux divergence from the photosphere and lower chromosphere is obtained by angle and wavelength integration of the transport equation. Assuming isotropic opacities $\chi_{\lambda}$ and emissivities we find

$$
\nabla \mathbf{F}_{r}=4 \pi \int_{\lambda} \epsilon_{\lambda} \chi_{\lambda}\left(B_{\lambda}-J_{\lambda}\right) d \lambda
$$

where $\epsilon_{\lambda}$ is the photon destruction probability. The method of solving the transport equation assumes opacities are in LTE and coherent scattering. Nordlund (1982) devised a technique of solution based on group mean opacities, in which the spectrum is divided into four bins representing strong, medium and weak lines in addition to the continuum. The transfer equation is formulated for these bins and a group mean source function is calculated for each bin. These source functions contain an approximate scattering term and an exact contribution from thermal emissivity. The resulting 3D scattering problems are solved by iteration based on a one-ray approximation in the angle integral for the mean intensity as developed by Skartlien (2000).

For the upper chromosphere and corona we assume optically thin radiative losses such that:

$$
\nabla \mathbf{F}_{r} \propto \rho^{2} f(T) e^{-\tau}
$$

where $f(T)$ is the optically thin radiative loss function based on the coronal approximation and atomic data collected in the HAO spectral diagnostics package (Judge \& Meisner 1994); $f(T)$ is based on the elements hydrogen, helium, carbon, oxygen, neon and iron. The $e^{-\tau}$ term prevents this term from having an effect in the deep photosphere, where we assume an "optical depth", $\tau$, proportional to the gas pressure.

In the mid and upper chromosphere we include non-LTE radiative losses from hydrogen continua, hydrogen lines and lines from singly ionized calcium. These losses are calculated from

$$
\nabla \mathbf{F}_{r} \propto N_{e} \rho f_{i}(T) \epsilon_{i}\left(m_{c}\right)
$$

where $N_{e}$ is the electron density, $f_{i}(T)$ is a pre-calculated function based on collisional excitation and radiative de-excitation, separate for hydrogen and calcium, and $\epsilon_{i}\left(m_{c}\right)$ is the non-LTE escape probability as a function of column mass $\left(m_{c}\right)$. This escape probability function is calculated from $1 \mathrm{D}$ dynamical chromospheric models in which the radiative losses are computed in detail (Carlsson \& Stein 1992, 1995, 1997, 2002). In addition to these 
optically thin losses in the upper atmosphere we have added an ad hoc heating term to prevent the atmosphere from cooling much below $2000 \mathrm{~K}$ in the upper chromosphere.

The Courant condition for a diffusive operator such as that describing thermal conduction scales with the square of the grid size $\Delta z^{2}$ instead of with $\Delta z$ as applies to the magneto-hydrodynamic operator. This severely limits the time step $\Delta t$ the code can be stably run at. Our solution to this problem is to proceed by operator splitting such that the operator advancing the variables in time is $L=L_{\text {hydro }}+L_{\text {conduction. }}$. Thus the conductive part of the energy equation is solved by discretizing.

$$
\frac{\partial e}{\partial t}=\nabla \mathbf{F}_{c}=-\nabla \kappa_{\|} \nabla_{\|} T
$$

using the Crank-Nicholson method and solving the resulting implicit problem using a multigrid solver. The formulation used in the code described here is based on the method used by Malagoli, Dubey, Cattaneo as shown at http://astro.uchicago.edu/Computing/On_ Line/cfd95/camelse.html, but extended to 3D and to confining conduction to follow the magnetic field lines.

The units employed in this works are in SI. Sometimes we refer the distance in Mm $\left(10^{6} \mathrm{~m}\right)$ and the time in hs $\left(10^{2} \mathrm{~s}\right)$.

\section{Initial and boundary conditions}

The five models described here are run on a grid of $256 \times 128 \times 160$ points spanning $8 \times 4 \times 16 \mathrm{Mm}^{3}$ and $16 \times 8 \times 16 \mathrm{Mm}^{3}$. At these resolutions the models have been run for roughly one hour solar time.

The average temperature at the bottom boundary is maintained by setting the entropy of the fluid entering the computational domain. The bottom boundary is otherwise open, allowing fluid to enter and leave as required. The upper boundary is set so that the temperature gradient is zero; no conductive heat flux enters or leaves the computational domain through the top boundary.

Of course, without coronal heating the corona would cool on a time scale of roughly an hour (depending on the magnetic field topology), when no heat flux enters the model through the upper boundary. We have therefore seeded the initial model with a magnetic field in which sufficient stresses can be built up to maintain coronal temperatures in the upper part of the computational domain, as previously shown to be feasible by Gudiksen \& Nordlund (2004). The initial field was obtained by semi-randomly spreading some $20-30$ positive 
and negative patches of vertical field at the bottom boundary, then calculating the potential field that arises from this distribution in the remainder of the domain. Stresses sufficient to maintain a minimal corona are built up by photospheric motions after roughly 20 minutes solar time.

At the top boundary the hydrodynamic variables (aside from the temperature) and the magnetic field are set by characteristic extrapolations. This method hinders most of the reflections that are due to the presence of the upper boundary. No joule heating is added in the top five computational zones.

\subsection{Initial model}

The initial atmosphere, into which magnetic field is injected, includes the upper convection zone, the photosphere, chromosphere, transition region, and corona. In the left panel of figure 1 we show the lower $12 \mathrm{Mm}$ (of $16 \mathrm{Mm}$ ) minimum, maximum, and horizontally averaged temperatures and densities as a function of height $z$. At the base of the model, 1.4 Mm below the photosphere, the average temperature is some $16200 \mathrm{~K}$ and average density $6.7 \times 10^{-3} \mathrm{~kg} \mathrm{~m}^{-3}$. With increasing height the temperature falls close to adiabatically up to the photosphere, which is located at $z \approx 0 \mathrm{Mm}$, where the temperature gradient becomes much steeper and the average temperature falls to $6000 \mathrm{~K}$. The average density falls roughly 1.5 orders of magnitude to $2.5 \times 10^{-4} \mathrm{~kg} \mathrm{~m}^{-3}$ in the same height range. Quantities vary much more in the chromosphere, which extends from the top of the photosphere up to a height of some $4 \mathrm{Mm}$ in some locations. The chromospheric temperature varies from a low of $2000 \mathrm{~K}$ up to almost $10000 \mathrm{~K}$ in the strongest shocks and in regions of strong magnetic field. The density also has a high horizontal contrast in the chromosphere while at the same time falling by $7-8$ orders of magnitude vertically. The location of the transition region varies from 1.5 to $4 \mathrm{Mm}$ above the photosphere. In this region the temperature rises rapidly

to coronal values of roughly $1 \mathrm{MK}$, while coronal densities are of order $10^{-12} \mathrm{~kg} \mathrm{~m}^{-3}$ in this model.

In the right panel of figure 1 we plot the minimum, maximum, and horizontally averaged gas and magnetic pressures as a function of height. The average gas pressure is $8.7 \times 10^{5} \mathrm{~Pa}$ at the bottom boundary while the average magnetic pressure at the same depth is much smaller at $1.6 \times 10^{3} \mathrm{~Pa}$. The ratio between these is still large in the photosphere, but note that the maximum magnetic pressure is as large as the maximum gas pressure here; photospheric motions can concentrate the magnetic field to pressures that equal or exceed photospheric pressure in intergranular lanes. The average plasma $\beta$ (ratio of gas pressure to magnetic pressure) remains greater than one up to some $600 \mathrm{~km}$ above the photosphere, but the $\beta=1$ 
plane is quite corrugated and can extend up to $z=1000 \mathrm{~km}$. This means that the upper chromosphere, transition region and lower corona all are low $\beta$ regions. In the initial model, there is little net magnetic flux and the field is small at great heights, we therefore find some high $\beta$ regions at heights greater than $z=8 \mathrm{Mm}$ or so.

The numerical box and the axes used are shown in figure 2, The color scale represents the temperature. The red lines are the magnetic field lines in the initial background field. Note that field lines extending into the corona are mainly rooted in regions that cover all $y$ values and around $x=7 \mathrm{Mm}$ and around $x=14 \mathrm{Mm}$. In addition we show planes of temperature at four heights: in the photosphere $z=10 \mathrm{~km}$, in the reverse granulation $z=234 \mathrm{~km}$, at the layer $z=458 \mathrm{~km}$, and in the chromosphere at $z=900 \mathrm{~km}$. We will show details of how the initial atmosphere reacts to flux emergence at these heights later in this paper. The green lines show the magnetic field lines of the flux tube coming in from the bottom boundary.

\subsection{Injection of magnetic flux}

We introduce a magnetic sheet or magnetic flux tubes into the lower boundary of the model described in section 3.1. The OSC uses a numerical method that, in principle, does not change $\nabla \cdot \mathbf{B}$ in time. The solenoidal condition must be enforced on the boundary to ensure that no magnetic monopoles are introduced. This is implemented by applying the boundary condition to the electric field, the staggered mesh will then enforce $\nabla \cdot \mathbf{B}=0$ to the numerical accuracy of the operators at the boundary.

The magnetic field variation at the boundary is defined by:

$$
\frac{\partial \mathbf{B}}{\partial t}=\nabla \times \mathbf{E}
$$

where, for example for the $x$ component of the electric field we have

$$
E_{x}^{n}=E_{x}+\frac{\Delta\left(B_{y}\right)}{\tau} \Delta z
$$

where $E_{x}^{n}$ is the new $x$ component of the electric field at the boundary, $\tau$ is the time step size, and $\Delta z$ is the vertical cell size, $\Delta\left(B_{y}\right)=B_{y}^{n}-B_{y}$ is the difference between the value of the magnetic field we would like to impose at the boundary, $B_{y}^{n}$, and the current boundary field $B_{y}$.

We have run models in which a constant horizontal field in the $y$ direction has been injected, and several models where a flux tube is introduced. The flux tube is horizontally 
rectilinear with twisted magnetic fields lines. The expression for the magnetic field has a structure given by

$$
\begin{aligned}
\mathbf{B}_{\text {long }} & =B_{o} \exp \left(-\frac{r^{2}}{R^{2}}\right) \mathbf{e}_{z} \\
\mathbf{B}_{\text {trans }} & =B_{\text {long }} r q \mathbf{e}_{\phi},
\end{aligned}
$$

where $r=\sqrt{\left(x-x_{o}\right)^{2}+\left(z-z_{o}\right)^{2}}$ is the radial distance to the center of the tube that has radius $R$. $\mathbf{B}_{\text {long }}, \mathbf{B}_{\text {trans }}$ are the longitudinal and transversal magnetic fields in cylindrical coordinates respectively. The parameter $q$ is used by Linton et al. (1996) and Fan et al. (1998) to define the twist of the magnetic field. Following Cheung \& Moreno-Insertis (2006), we define a new twist parameter, $\lambda$, as

$$
\lambda \equiv q R
$$

As the flux tube enters the computational box, the height of the center of the tube $\left(z_{o}\right)$ changes in time. The speed of flux tube, $\left(d z_{o} / d t\right)$, is set to the average of the velocity of plasma inflow at the boundary in the region where the magnetic flux tube is located each time step.

The field defined by equations 11 and 12 is easily seen to be a horizontal axisymmetric magnetic flux tube in which the longitudinal field has a gaussian profile in the radial direction.

\section{Results}

We have carried out five simulations with different twist and magnetic field strength in order to study the effects of flux emergence in the photosphere and in the chromosphere. A summary of the runs completed is shown in table 1. In describing the reaction of the atmosphere to the introduction of new magnetic flux we will specifically concentrate on four layers placed at heights of $z=10,235,450$, and $900 \mathrm{~km}$ represented in the figure 2 .

We find that all runs show a similar series of events after the emerging magnetic flux pierces the photosphere. These events, that occur at roughly the same time in all models, are summarized in table 2, which shows the processes observed in the simulations ordered in time. These processes are described in the following sections according to where they happen. 
In the following sections all the figures shown correspond to simulation A4 unless otherwise noted, both because it has run for a long solar time $(T=3200 \mathrm{~s})$ and as it has the most pronounced processes as a result of flux tube emergence.

\subsection{Photosphere}

The photosphere is located at $z \approx 0 \mathrm{Mm}$ and is, up to the time when the emerging flux penetrates, as described in section 3.1, with a cellular temperature structure similar to that shown in the upper left panel of figure 3. As the flux tube approaches the photosphere from below the cells immediately above the flux tube expand as seen in the upper right panel of figure 3. Eventually, horizontal field pierces the photospheric surface in the granular cell centers as seen in the second panel of the right column of figure 4. The horizontal field is rapidly moved to the intergranular lanes as a result of the granular flow (figure 4 third row). The granular cells where the field penetrates remain larger than in the undisturbed state as the flux tube passes through the photosphere. At some point, the expansion lowers the temperature of the expanding cells through adiabatic cooling. At later stages a small amount of the magnetic flux that pierces the photosphere is returned to the convection zone. The photospheric evolution of emerging flux as described above has already been extensively studied previously and reported in the literature e.g. Cheung et al. (2007) and references cited therein.

In all the simulations we find that approximately $2100 \mathrm{~s}$ passes from the time the magnetic flux tube crosses the photosphere until the time the photosphere again appears to regain its "normal" state, i.e. until the photospheric again is similar to the initial state before the flux tube passes through (see figure 3). Let us study the figures in more detail.

In figure 5 we show two 3D images of the A4 simulation from two different viewpoints; from above and from below the photosphere as seen at time $t=1700 \mathrm{~s}$. The figure shows how the temperature structure of the granular cells has been changed by the presence of the flux tube, and in addition how the structure of the flux tube itself has been modified. The tube has suffered an expansion and splitting, gaining an irregular structure due to the movements of the convective cells.

The magnetic field evolution in the photosphere is shown in the figure 4. The maximum magnetic field-strength in the photosphere varies between $850 \mathrm{G}$ and $1060 \mathrm{G}$. However, during the first hectosecond (hs) after the flux tube enters the photosphere the maximum rises to

between $970 \mathrm{G}$ and $1030 \mathrm{G}$. Later, the magnetic field is concentrated by the granular flow and the maximum rises to some $1100 \mathrm{G}$. The increased maximum magnetic field-strength is 
observed from $1700 \mathrm{~s}$ until the simulation ends, in some instants rising to $1700 \mathrm{G}$ during the last few hectoseconds of the simulation. The magnetic field is almost horizontal and centered in the cells when the flux tube starts to cross the photosphere. The magnetic field is confined by the fluid and displaced to cell edges at which time its orientation becomes vertical. After most of the original tube has crossed the photosphere, some remaining magnetic flux that has remained below the photosphere appears. This remaining flux is small compared to the main tube's emergence and appears far away on either side of the central tube axis. The emergence process for this remaining flux is similar to the main tube's emergence, in that almost horizontal field rises in the cell center to be subsequently transported to the intergranular lanes where it becomes vertical as also reported earlier by Cheung et al. (2007) (see the last row of figure 4).

The intensity in the opacity bin with smallest opacity, designed to mimic the continuum, is shown in figure 3 along with contours of the magnetic field strength at height $z=10 \mathrm{~km}$ at 6 different instants in time; at 600, 1100, 1210, 1660, 2200, and $3220 \mathrm{~s}$. The evolution of the intensity is very similar to that of the temperature: Granular cells, located where the flux tube emerges, become larger and darker as they expand. These large cells cool as their size increases due both to radiative looses and to adiabatic expansion. After the tube has crossed the photosphere the granulation pattern returns to normal, roughly at times greater than $2100 \mathrm{~s}$.

The temperature range before the tube crosses the photosphere is $[6.0,10.2] \mathrm{kK}$. While the upper value of the photospheric temperature remains the same, the lower limit varies: when the tube is just below the photosphere it rises to $6.2 \mathrm{kK}$, thereafter decreasing to $5.6 \mathrm{kK}$ as large granulations cells cool by expansion (at time $1300 \mathrm{~s}$ ). In the latter stages of the simulation the lower temperature stabilizes at $5.8 \mathrm{kK}$.

The range in vertical velocity is roughly $[-6,5.5] \mathrm{km} \mathrm{s}^{-1}$ where positive means upwards flow. It is well known that downflows in the intergranules are faster than the upward granular flow. However, it appears that the maximum and minimum vertical velocity increase after time $1100 \mathrm{~s}$, i.e. during and after the tube crosses the photosphere. The maximum upward velocity increases to $8 \mathrm{~km} \mathrm{~s}^{-1}$ when the tube is in the photosphere, i.e. from $1100 \mathrm{~s}$ to $1600 \mathrm{~s}$. Later the maximum returns to normal values, but still shows large variations in time. The maximum horizontal velocity oscillates between $[6,7] \mathrm{km} \mathrm{s}^{-1}$ except at time $1350 \mathrm{~s}$ when there is a big jump to $10 \mathrm{~km} \mathrm{~s}^{-1}$, that lasts until $t \approx 2000 \mathrm{~s}$. We do not see any remarkable patterns in the extrema of vertical velocity before, during or after the tube crosses the photosphere.

It is worth remarking that we see several examples of collapsed cells (see Skartlien et al. 2000) visible near the boundary of the flux tube as it crosses the photosphere (see the topright at time $1100 \mathrm{~s}$ and bottom left at time $1210 \mathrm{~s}$ panels at $x \approx 3 \mathrm{Mm}$ and at $x \approx 5 \mathrm{Mm}$, 
the blue line of the figure (3). Squeezing of the granule occurs as the adjacent plasma expands during the rise of the flux tube and by the tendency for downflows surrounding a small granule to merge in the boundary layers. There are also bright points visible after the tube crosses the photosphere, as seen in the lower left panel of the figure 3 at position $(x, y) \approx(6.5,2.7) \mathrm{Mm}$ at time $2200 \mathrm{~s}$ or the bottom-right panel at the position $7,3.8 \mathrm{Mm}$. At that position the temperature is low at photospheric height, but the magnetic field is strong and the decreased gas density (and thus photospheric opacity) allows us to see deeper into the atmosphere where the temperature is higher (see also Shelyag et al. 2004; Carlsson et al. 2004).

In figure 6 we show the evolution of the mean of magnetic flux of simulations A1, A2, A3 and A4 by area. We define the mean of the magnetic flux per unit of area between 2 heights by:

$$
<\phi>=\int_{0}^{L}\left(\int_{0}^{L_{h}} \int_{l}^{u} \mathbf{B} d \mathbf{S}\right) d n /(L|\mathbf{S}|)
$$

where $\phi$ is the mean of magnetic flux integrated in the area in the vertical plane between $z=l$ and $z=u$ in the vertical component of $\mathbf{S}$,i.e. $L_{z}=u-l$ and along the whole horizontal component of $\mathbf{S}$, i.e. from 0 to $L_{h}$. The mean is carried out along the perpendicular component of $\mathbf{S}$, i.e. it is defined as the integral over the line perpendicular to the surface $\mathbf{S}$ divided by the length in that direction of the box domain. We are interested in measuring the flux in the $y$ direction, then the expression 14 simplifies to:

$$
<\phi>=\int_{0}^{L_{y}}\left(\int_{0}^{L_{x}} \int_{l}^{u} B_{y} d z d x\right) d y /\left(L_{y} L_{x} L_{z}\right)
$$

where $L_{x}$ is the length of the box in the $x$ direction, $L_{y}$ is the length in the $y$ direction and the integration in $z$ goes from $z=l$ to $z=u$. We consider two different integrated regions in the figure 6, one is below the photosphere $(z<0.01 \mathrm{Mm})$, i.e. from $l=-1.5 \mathrm{Mm}$ to $u=0.01 \mathrm{Mm}$, in red color, and the second from $l=0.01 \mathrm{Mm}$ to $u=245 \mathrm{~km}$, in black. The figure shows that the greater the twist, the greater the amount of magnetic flux that crosses the photosphere. On the other hand, the time needed for the tube to cross the photosphere is similar in all simulations. (It should be kept in mind that there is also magnetic flux being lost through the bottom boundary of the simulation box in addition to that rising beyond the photosphere into the upper atmosphere.) The simulation A2 (with weaker injected field $B_{o}$ ) has a smaller total flux and the slope $(d\langle\phi\rangle / d t)$ is much smaller than in the other simulations. When the tube has finished crossing the photosphere $(t>2000 \mathrm{~s})$ the flux retained above the photosphere is in quasi-equilibrium and oscillates with a period of roughly 5 minutes. 
This oscillation is due in part to the magnetic field that is returned to the photosphere and in part because the direction of the tube changes in time. The oscillation in flux strength coincides with oscillations in maximum downward velocity variation with time, but there is a small delay of around $30 \mathrm{~s}$ between the flux and the velocity variation. We find the same phenomenon in upper layers (see section 4.2 and 4.3).

The average amount of magnetic flux in the convection zone is greater than in the photosphere, which means not all of the flux tube crosses into the photosphere. The only portion of the magnetic tube that reaches the photosphere is the part that has a steeply decreasing field strength with height. Cheung et al. (2007) have pointed out that the higher the level of twist in the initial flux tube, the larger the fraction of magnetic flux that can pass into the regions above the photosphere. This can be explained as a consequence of the magnetic tension of the transverse fields tending to keep the tube coherent as shown by Moreno-Insertis \& Emonet (1996). Following the linear stability analysis of Acheson (1979) we find that the photosphere has a superadiabatic excess profile, defined by $\delta_{T}=\nabla-\nabla_{a d}$, that is shaped like a gaussian with height. In this case the magnetic buoyancy instability sees a barrier in the photosphere and the emerging tube velocity decreases as it comes closer to the photosphere. Only the portion of the flux tube where the gradient of the magnetic field strength is larger than the superadiabatic excess in the photosphere can reach the layers above the photosphere. The relation between the buoyancy instability and the superadiabatic excess has been studied by several authors (Acheson 1979; Magara \& Longcope 2001; Archontis et al. 2004).

The field left behind after the tube has passed is more vertical than it was before the tube passes through a given particular height. This behavior is more evident near the end of the simulation where the magnetic field is more vertical in the chromosphere. The ratio between $B_{z}$ and $|B|$ oscillates in time in much the same manner as does the maximum downward velocity in the photosphere between $z=0 \mathrm{~km}$ and $z=458 \mathrm{~km}$.

\subsection{Reverse granulation}

Intensities originating a few hundred kilometers above the granulation show a pattern that is reverse that of granulation, with low intensity above granules and higher intensity above intergranular lanes (Evans \& Catalano 1972; Suemoto et al. 1987, 1990; Rutten \& Krijger 2003). The reason for the inverse contrast is the cooling from a divergent velocity field above granules and heating from a convergent velocity field above the intergranular lanes. The final intensity pattern from this layer may also be influenced by internal gravity waves but does not appear to be related to the magnetic field (Rutten et al. 2004; Leenaarts \& Wedemeyer-Böhm 
2005). However, as we discuss below, when the magnetic flux crosses this region, the contrast and the structure of the reverse granulation is modified.

Figure 7 shows 3D images in two different views at $t=1900 \mathrm{~s}$; as seen from above and from below the reverse granulation layer. The figure shows how the reverse granulation has been changed by the presence of the rising magnetic flux tube and is colder in the interior of the reverse granulation cells in regions where the tube is located than outside. Also at this height we can see how the structure of the tube has been disrupted as it has passed through the atmosphere. In fact, the structure of the tube is more split and fragmented than that seen in figure 5 .

We define a horizontal layer at $z=234 \mathrm{~km}$. At this height the granular pattern is still clearly visible, but it is reversed compared to the photosphere (figure 8). We see expansion of the cells beginning at time $920 \mathrm{~s}$. The flux tube starts to go through the reverse-granulation layer some $80 \mathrm{~s}$ after it crosses the photosphere, i.e. at time $1380 \mathrm{~s}$. As in the photosphere, the reversed granules increase in size as the flux tube approaches. The magnetic field lines of the tube are seen in figure 7 to go from one side of the expanded cells to the other.

Figure 9 shows the evolution of the vertical magnetic field $B_{z}$ in the left column and the horizontal magnetic field in the right column, at three different instants in time. The maximum magnetic field-strength, before the flux tube reaches the reverse granulation, is of order $500 \mathrm{G}$. When the tube starts to cross the height $234 \mathrm{~km}$ the maximum rises to $600 \mathrm{G}$. At later times, but while parts of the tube are still to be found at this height, the remaining magnetic field is confined to downflow regions and the maximum field strength rises to $650 \mathrm{G}$. After the tube has passed, some remaining confined flux rises to a maximum of up to $800 \mathrm{G}$. During the last $500 \mathrm{~s}$ of the simulation the maximum field strength oscillates around $700 \mathrm{G}$. Again, as in the photosphere, the magnetic field is initially horizontal and appears in the center of the granular cells (top panels). The magnetic field is then confined and moves with the fluid to the edges of the cells where it becomes vertical (see the second and third rows of the figure 9). At the cell edges a great number of bipoles form by $t=2100 \mathrm{~s}$. However, at later times the bipoles are canceled by magnetic diffusion; i.e. in the bottom panel on the left side of figure 9 we find generally that negative (downward) magnetic field is concentrated while we find positive (upward) flux on the right.

The top row of figure 8 shows the continuum intensity at $170 \mathrm{~nm}$ as calculated from the simulation at times $1620 \mathrm{~s}$ (left) and $3220 \mathrm{~s}$ (right). The continuum opacity at this wavelength is dominated by Fe bound-free opacity and the monochromatic optical depth unity is around $250 \mathrm{~km}$ height in the simulation. Note that real observations around this wavelength (such as the TRACE $170 \mathrm{~nm}$ bandpass) are dominated by line opacity, with the intensity thus coming from higher layers. The intensity images shown here are, however, typical of a diagnostic 
formed in the reverse granulation layer (like the inner wings of the resonance lines from ionized calcium). The figure also shows the vertical velocity in the second row with the same grey-scale values, i.e. from $2.6 \mathrm{~km} \mathrm{~s}^{-1}$ (white) to $-4.1 \mathrm{~km} \mathrm{~s}^{-1}$ (black), the divergence of the velocity in the third row and the vertical vorticity in the fourth row, all these variables at height $234 \mathrm{~km}$. The synthetic $170 \mathrm{~nm}$ intensity shows reverse granulation and it is clear that at $t=1620 \mathrm{~s}$ the granular cells have expanded in regions co-spatial with the flux tube. The same pattern is also found in plots of the temperature and the vertical velocity. Larger cells grow dimmer and colder due to the expansion as can be verified by considering the velocity divergence. While cool, dim regions are due expansion the hotter bright network points are seen to be related to contraction of the fluid at this height. At the end of simulation we find several bright points. This is seen clearest at time $t=3220 \mathrm{~s}$ in the regions where we find concentrated vertical magnetic field (see second column of the figure 8 at the position $[6.7,3.7] \mathrm{Mm})$. These bright points in intensity are related to large vertical velocities, but also to high temperatures compared to the surroundings, a large vertical vorticity and to compression of material (black color in the divergence of the velocity plots). The heating process for these bright points is clearly related to the high velocity convergence and high vorticity. We will find similar bright points at greater heights in the atmosphere, though with larger horizontal extent.

The temperature range is found to be [4.9,6.2] kK before the tube reaches the $234 \mathrm{~km}$ layer, while during and after the flux tube passes the temperature range is $[4.6,6.1] \mathrm{kK}$; the lower limit decreases in time after $2400 \mathrm{~s}$. The reason for this temperature drop is the expansion as discussed above.

There are no large changes in the upward maximum velocity with time before, during or after the tube crosses the layer $234 \mathrm{~km}$ height; it is found to be around $2.5 \mathrm{~km} \mathrm{~s}^{-1}$. The maximum downward velocity does not change until after $t=1400 \mathrm{~s}$ when it has a small increase for $200 \mathrm{~s}$. The maximum downward velocity then returns to normal before increasing from $3 \mathrm{~km} \mathrm{~s}^{-1}$ to $5 \mathrm{~km} \mathrm{~s}^{-1}$ starting at $t=2200 \mathrm{~s}$. These large downflow velocities are found to correspond to the small regions with a strongly confined magnetic field in the intergranular lanes. Examples of such a regions are found in the second row, right column, of figure 8 at position $[x, y]=[4.1,0.2] \mathrm{Mm}$ and also at $[7.1,3.6] \mathrm{Mm}$. Towards the end of the simulation, at time $3100 \mathrm{~s}$, the maximum downward velocity has returned to $3 \mathrm{~km} \mathrm{~s}^{-1}$. The maximum horizontal velocity oscillates around $5.5 \mathrm{~km} \mathrm{~s}^{-1}$. However, it has a peak at $1480 \mathrm{~s}$ with a maximum velocity of $8 \mathrm{~km} \mathrm{~s}^{-1}$ as the tube is crossing the layer $234 \mathrm{~km}$ height.

The expansion of the cells is observed at time $t=900 \mathrm{~s}$ in the photosphere $(z=10 \mathrm{~km})$ and roughly $25 \mathrm{~s}$ later in the reverse granulation layer $234 \mathrm{~km}$ height. The separation between these two layers is $224 \mathrm{~km}$. The propagation speed of the tube itself is much slower, as table 
2 shows, it spends $250 \mathrm{~s}$ in moving from the layer at $10 \mathrm{~km}$ to the layer at $234 \mathrm{~km}$ height. Another possibility for explaining the expansion of the cells at the layer $234 \mathrm{~km}$ height is by the propagation of Alfvén waves, but again, the time such waves need to propagate from the layer $10 \mathrm{~km}$ to the layer $234 \mathrm{~km}$ height is too long, because the mean Alfvén speed is very small in that region $\left(<100 \mathrm{~m} \mathrm{~s}^{-1}\right)$. On the other hand, the average sound speed between $10 \mathrm{~km}$ to $234 \mathrm{~km}$ height is $8.5 \mathrm{~km} \mathrm{~s}^{-1}$, which gives a travel time close to the $25 \mathrm{~s}$ that fits with the time to move outward the horizontal expansion of the cells. In other words we see the expansion of the granular cells some $400 \mathrm{~s}$ before the magnetic tube actually emerges through the photosphere, when the tube is situated $182 \mathrm{~km}$ below the photosphere. The speed of the sound below the photosphere is on average $10 \mathrm{~km} \mathrm{~s}^{-1}$ which means the expansion wave is produced roughly $420 \mathrm{~s}$ before the tube reaches the photosphere, i.e when the tube is roughly at $-246 \mathrm{~km}$. The average tube velocity from $-246 \mathrm{~km}$ to $10 \mathrm{~km}$ is $0.5 \mathrm{~km}$ $\mathrm{s}^{-1}$. However, the tube decelerates when it approaches the photosphere as explained in the section 4.1. The cells average horizontal expansion velocity is roughly $2.5 \mathrm{~km} \mathrm{~s}^{-1}$ before the tube reaches the photosphere.

The figure 10 shows the average magnetic flux in the same manner as in figure 6, but here the regions considered are from the layer $z=10 \mathrm{~km}$ to the layer $z=234 \mathrm{~km}$ (black color) and from the layer $z=234 \mathrm{~km}$ to the layer $z=458 \mathrm{~km}$ (red color). Each of the four displayed simulations conserve magnetic flux in the sense that when the magnetic flux tube crosses these regions no flux is lost or retained in the region, as opposed to what happens in the convection zone. The biggest difference between the two regions is seen to be in the slope of the flux $(d\langle\phi\rangle / d t)$ as the tube moves through the region. The slope of the flux is much smaller than that found in figure [ when the tube crosses those layers, i.e. more time is spent in crossing each region. The oscillation of the mean magnetic flux occurs more or less at the same time, when the tube crosses the corresponding region, but the amplitude of the variation is greater in higher regions.

\subsection{End of the photosphere-beginning of the Chromosphere}

The top of the convection zone, photosphere, and lower-chromosphere are the regions where acoustic waves are excited by convective motions. While the waves propagate upwards, they steepen into shocks, dissipate, and deposit their mechanical energy as heat in the

chromosphere. In studying the quiet non-magnetic chromosphere Wedemeyer et al. (2004) corroborates in 3D models the finding by Carlsson \& Stein (1994) that the chromospheric temperature rise derived from semi-empirical modeling of the time average intensity does not necessarily imply an increase in the average gas temperature but rather can be explained by 
the presence of substantial spatial and temporal temperature inhomogeneities, such as those caused by non-linear waves. We are interested in finding how the rise of a flux tube affects chromospheric dynamics and energetics, i.e. if there are other energy sinks or sources that become active with the appearance of new magnetic flux, or if the new flux modifies the propagation of acoustic waves into the middle and high chromosphere.

In our simulations the magnetic flux tube enters the height $z=458 \mathrm{~km}$ some $300 \mathrm{~s}$ after it has crossed the photosphere. The layer at $458 \mathrm{~km}$ height suffers various perturbations in response to the appearance of the flux tube. Of the most important effects is the expansion and the cooling related to that expansion. Each of these processes are explained below.

Figure 11] shows two 3D images of the A4 simulation at $t=1980 \mathrm{~s}$ from above and from below a temperature slice made at height $z=458 \mathrm{~km}$ above the photosphere. The temperature structure at that height $(z=458 \mathrm{~km})$ has been changed by the presence of the tube and is much cooler in regions where the tube is located. However, on the borders of the rising tube we find regions with higher temperatures. As in the previous 3D images, featuring lower heights, we see how the structure of the magnetic flux tube has been perturbed as a result of rising through the atmosphere; comparison with these figures shows the structure of the tube is at this late time even more splintered and fragmented than at earlier times (see figures 5 and 7 ).

The vertical magnetic field is shown in the left column of figure 12 and the horizontal magnetic field in the right column, both for 2 different instants at height $z=458 \mathrm{~km}$. The maximum magnetic field strength before the magnetic flux tube reaches this height is $170 \mathrm{G}$. During the flux emergence process the maximum field strength increases to $270 \mathrm{G}$, and at later times, as the magnetic flux is compressed and confined to smaller regions, the field strength in some points increases to $350 \mathrm{G}$. At this height there is a cellular structure at any given time, but the cells do not directly correspond to the photospheric granular cells, but rather to patterns in the velocity field that change from instant to instant. The vertical magnetic field is mostly confined to downflow regions and dominates the field. However, as the flux tube crosses the layer at $458 \mathrm{~km}$ height the horizontal magnetic field is strongest and fills the upflow cells. There are some regions or "points" with strong vertical magnetic field. For instance the bottom left panel of the figure 12 at position [7,4] Mm. These points have increasing maximum magnetic field-strength with time after the flux tube has passed through the layer and they move around with the fluid.

Figure 13 shows the calculated continuum intensity as described in the figure at $160 \mathrm{~nm}$ and $130 \mathrm{~nm}$ for two snapshots of the simulation. These images show what could be observed in the TRACE $160 \mathrm{~nm}$ filter and in a SUMER raster at $130 \mathrm{~nm}$. At both wavelengths the continuum opacity is dominated by bound-free opacity from silicon: at $160 \mathrm{~nm}$ from the 
first excited level and at $130 \mathrm{~nm}$ from the ground state. The monochromatic optical depth unity is on average around $480 \mathrm{~km}$ and $790 \mathrm{~km}$ height in the simulation at $160 \mathrm{~nm}$ and $130 \mathrm{~nm}$ respectively (in the VALC model atmosphere the corresponding heights are $456 \mathrm{~km}$ and $735 \mathrm{~km}$, respectively). The intensity at $t=1620 \mathrm{~s}$ shows expansion of the plasma with subsequent dimming, somewhat more clearly at $160 \mathrm{~nm}$. At $3220 \mathrm{~s}$ both the continua show a bright point at position $[7,3.8] \mathrm{Mm}$. It is also possible that the tube expansion could be affected by the finite horizontal size of the computational domain; figure 12 shows that the horizontal magnetic field almost reaches the boundary layers of the box. On the other hand the (limited number of) models we have run with a larger box, $16 \times 8 \times 16 \mathrm{Mm}^{3}$ do not show a fundamentally different behavior, so we believe the effects of limited simulation size are minor.

The vertical velocity is shown in figure 14 in the first row of panels using the same grey-scale intensity, from $1.5 \mathrm{~km} \mathrm{~s}^{-1}$ (white) to $-5.88 \mathrm{~km} \mathrm{~s}^{-1}$ (black); the divergence of the velocity in the second row and the vertical vorticity in the last row of panels, all at time $1620 \mathrm{~s}$ in the first column and at time $3220 \mathrm{~s}$ in the second column. We find similar structure to those found in the synthetic observations (figure 13) before the tube crosses the layer at $458 \mathrm{~km}$ height. The structure of the cells is strongly modified as the tube goes through that layer. We find that the cell expansion is related to the divergence of the velocity, also at this height, but that the correspondence between temperature/continuum emission and velocity divergence is not as clear as in the lower layers. There are large vertical velocities (first row of the figure 14), both up or down (see the left-panels of the figure 14 at position [2.1, 0.8] and right panels at position [7,3.7]) which correspond to the bright points in the synthetic observations and also fit with the large concentration of magnetic flux and vertical vorticity.

The maximum upward velocity oscillates around $1.5 \mathrm{~km} \mathrm{~s}^{-1}$ with no important changes in time except at $t=2420 \mathrm{~s}$ when the peak rises to $4 \mathrm{~km} \mathrm{~s}^{-1}$. This peak is due to the rapid movement of the tube as it rises. The maximum downward velocity oscillates around $2 \mathrm{~km}$ $\mathrm{s}^{-1}$. Later, the maximum downward velocity increases slowly with time from $t=1800 \mathrm{~s}$, up to $5 \mathrm{~km} \mathrm{~s}^{-1}$ towards the end of the simulation at $t=3220 \mathrm{~s}$. The downflowing plasma is located in the same region as the bright points with high magnetic field strength and vorticity are found. The maximum horizontal velocity varies around $3.5 \mathrm{~km} \mathrm{~s}^{-1}$ in the period from $0 \mathrm{~s}$ to $1400 \mathrm{~s}$. After that time it increases with time and rises to $7 \mathrm{~km} \mathrm{~s}^{-1}$ at time $1940 \mathrm{~s}$ due to the expansion process. Afterwards the maximal upward velocity returns to roughly $5.5 \mathrm{~km} \mathrm{~s}^{-1}$, though with big variations.

The temperature range before the tube crosses the layer $z=458 \mathrm{~km}$ is $[4,6] \mathrm{kK}$ from $0 \mathrm{~s}$ to $1300 \mathrm{~s}$. As the tube approaches the lower limit decreases to $3 \mathrm{kK}$ due to expansion. When the tube arrives, the temperature range changes to [3.2,6.2] kK at time $1900 \mathrm{~s}$. The 
lower limit increases to $3.9 \mathrm{kK}$ after $2500 \mathrm{~s}$.

After the magnetic flux tube has crossed the layer at $458 \mathrm{~km}$ height the structure of the plasma no longer appears granular. The magnetic field, shown in the figure 12, is almost horizontal and only in the boundary layer of the tube can one see any vertical component. With greater initial twist the tube remains more horizontal in the chromosphere compared to the cases with less initial twist, i.e. the ratio of the horizontal magnetic field component to the vertical magnetic field component, in regions where the tube is located, increases with increasing twist.

Figure 14 also shows the expansion of the fluid as the tube crosses the layer at $458 \mathrm{~km}$ height. The center of these cells are colder than the edges as the tube expands. However, as the tube crosses the layer at $458 \mathrm{~km}$ height, the small cells seen in the initial model disappear and cold larger zones form near the center of the tube. This is presumably due to expansion (second row of the figure 14), but the velocity divergence does not fit as well with the temperature or intensity maps as found in the lower layers of the height $458 \mathrm{~km}$. This suggests that at this height also other processes could be active in cooling the chromosphere. We will go into more detail on these alternate processes in the next section, as they become steadily more important with increasing height into the chromosphere.

At the boundary layer of the magnetic flux tube the temperature rises. This heating could be due to a wave, compression of the plasma, or reconnection where the magnetic field is almost vertical. Once the tube has disappeared from the layer at $458 \mathrm{~km}$, at time $t \approx 2300 \mathrm{~s}$, the temperature displays an irregular structure with some high temperature points. These points are coincident with a high vertical vorticity and with strong vertical magnetic field (e.g. at position [7,3.8] Mm at time $3220 \mathrm{~s}$, see figures [12-14). Note that the temperature structure has not returned to its pre emergence state. This could be due to the fact that the magnetic structure of the atmosphere has changed: before the tube appeared the energy density of the magnetic field was low and $\beta>1$, but around $t=2200 \mathrm{~s}$ we find several regions where $\beta \approx 0.5$ and the field dominates the chromospheric structure.

Bright points are seen to occur in the synthetic observations (figure 13). In the layer at height $458 \mathrm{~km}$ the bright points have greater contrast than found in the regions below and also much greater temperature than in the surrounding plasma (The bright point in figure 13 right-top panel at the position $[7,4] \mathrm{Mm}$ has a temperature at this height of $6500 \mathrm{~K}$ compared with the surrounding temperature of $5100 \mathrm{~K}$. At the same position at height $234 \mathrm{~km}$ (see figure (8) the temperature is $5700 \mathrm{~K}$ compared with $5200 \mathrm{k}$ in the surroundings) As explained earlier for the lower layers, bright points are related to strong magnetic field, to vertical velocity, and vertical vorticity, and to a larger compression 
than in the surroundings. The heating seems to come primarily from the convergence of the velocity field as we find the most important heating term is the advection term; it is an order of magnitude greater than the joule and viscous heating terms.

Figure [15] shows the total flux for the regions from $z=234 \mathrm{~km}$ to $z=458 \mathrm{~km}$ in red, and from $z=458 \mathrm{~km}$ to $z=905 \mathrm{~km}$, in black. The biggest difference between the figures [6, 10 and 15] is the slope of the flux with time $(d\langle\phi\rangle / d t)$. The total flux entering the chromosphere is much smaller than that reported in figures 6] and 10 because the tube spends more time in crossing the upper regions. This is presumably due to the horizontal expansion of the magnetic flux tube. The amplitude of the slope of the curves in the figure 15 increase with magnetic field strength. After the initial large rise, when the tube crosses the corresponding section, the curve shows an oscillation (see figure 15) that is quite similar to that seen in the layers below (see figures 6 and 10). However, by comparing each curve it is possible to see that there is a small delay between the different lines in the figures 6 , 10 and 15. This retardation of the oscillation between the different layers is what we would expect from a wave that propagates at the sound speed between each layer. The variation of the mean magnetic flux strength increases with increasing height, twist, and/or the magnetic field strength. The oscillations seem related to the oscillations in the downward maximum velocity with time and also to oscillations in the ratio between the vertical and horizontal magnetic field, as mentioned in the previous sections.

Finally, we find that the slope of the flux with time, $d\langle\phi\rangle / d t$, at the time the tube crosses into the chromosphere, is larger with greater values of the twist parameter and with larger magnetic field strength. This effect is clearer in the chromosphere than it is in the photosphere. Previous 2D flux emergence simulations by Shibata et al. (1989) and Magara \& Longcope (2001) have also identified magnetic buoyancy instabilities as important in the rise of magnetic field to the chromosphere and coronal layers.

\subsection{Chromosphere, Transition Region and Corona}

Let us finally discuss the effects of the magnetic flux tube as it reaches the upper chromosphere, transition region and lower corona. The variation of the magnetic field in the chromosphere, at $z=906 \mathrm{~km}$, is shown in figure 16 with the vertical field in the left column and the horizontal field in the right column, both at three instants; times $600 \mathrm{~s}, 2800 \mathrm{~s}$ and $3220 \mathrm{~s}$. The first signs of the magnetic flux tube reaching the chromosphere are evident at time $t=2100 \mathrm{~s}$. However, the tube does not appear uniformly in the region from $x=2 \mathrm{Mm}$ to $x=6 \mathrm{Mm}$ as it does in the levels below. Rather patches of magnetic flux rise into different places randomly along the tube region at different times. The maximum absolute magnetic 
field, before the tube reaches the layer is $43 \mathrm{G}$. This value increases after the tube comes into the chromosphere to $80 \mathrm{G}$. During the flux emergence into this level and after the tube has passed the average of the maximum field is of order of $65 \mathrm{G}$. At this height the magnetic field does not have any singular isolated points with strong amplitude. The field becomes generally more horizontal once the tube crosses this layer and has a smoother structure than in the layers below.

In figure 17 we show the intensity of the $\mathrm{C}$ I continuum at $109.9 \mathrm{~nm}$ (as could be observed with SUMER). We have calculated intensities at $t=1620 \mathrm{~s}$ before the emerging magnetic flux has entered the chromosphere and at $t=3220 \mathrm{~s}$, some 15 minutes after the emerging flux first appears. The mean monochromatic optical depth unity is around $1100 \mathrm{~km}$ height in the simulation of the $\mathrm{C}$ I continuum at $109.9 \mathrm{~nm}$ (in the VALC atmosphere model it is at $z \approx 975 \mathrm{~km}$ ). We also show synthetic Hinode observations of the Ca II H-line at times 600, 1620, 2110 and 3220 s. Note that while the carbon continuum is formed mainly in the chromosphere the Ca II as observed with the $0.22 \mathrm{~nm}$ wide Hinode filter has contributions from the entire atmosphere, stretching from the upper photosphere to the chromosphere (Carlsson et al. 2007). The structure of the intensity formed at this height shows large changes before and after the magnetic flux tube reaches the chromosphere. The temperatures range in the chromosphere at height $906 \mathrm{~km}$ before the tube reaches this level is $[2.2,7.2] \mathrm{kK}$, while after the tube has reached this height the range is $[2.0,7.0] \mathrm{kK}$. However, note especially the large dim (and cold) zones that become evident after the tube crosses into the chromosphere at time $3000 \mathrm{~s}$. These dim, cold, regions are much larger than in the lower layers (see section 4.2 and 4.3).

It is worth remarking that we do not find the concentrated bright points that appear in the lower lying layers. This is presumably because the magnetic field is mainly horizontal and we do not observe the confinement of the magnetic field that occurs in the regions below due to plasma motions. On the other hand, the chromosphere and corona suffer large changes in size, structure and temperature after the tube enters this zone. The cooling in the layers between $234 \mathrm{~km}$ and $458 \mathrm{~km}$ height is due to radiative losses and superadiabatic expansion, but in the chromosphere the big cold regions do not fit as well with the local plasma expansion as in the lower layers.

The vertical velocity in the chromosphere at height $906 \mathrm{~km}$ is shown in figure 18 at four different instants; 600, 2110, 2800 and $3220 \mathrm{~s}$. The maximum downward velocity is around $10 \mathrm{~km} \mathrm{~s}^{-1}$ and the maximum upward velocity is around $7 \mathrm{~km} \mathrm{~s}^{-1}$ throughout the whole simulation. It is difficult to see any effects due the emergence of flux in the maximum horizontal velocity as these have large changes with time. The average maximum horizontal velocity is $13 \mathrm{~km} \mathrm{~s}^{-1}$ and varies between $7 \mathrm{~km} \mathrm{~s}^{-1}$ and $20 \mathrm{~km} \mathrm{~s}^{-1}$. On the other hand the 
structure of the velocities change radically as is evident by comparing the pre- and postemergent panels of figure 18. Shock waves dominate the upper chromosphere before the tube crosses the chromosphere $(t=600 \mathrm{~s})$. However, during and after the tube crosses the chromosphere the velocity is much smoother in the regions where the field strength increases due to the rise of the flux tube into to upper chromosphere.

That the chromosphere and overlying transition region and lower corona suffer large structural changes as a result of flux emergence is driven home by considering a vertical slice of the atmosphere as shown in the left panel of figure 19. The transition region has been pushed by the emerging flux from a height that varies with chromospheric dynamics from around $2 \mathrm{Mm}$ to almost $6 \mathrm{Mm}$. This panel also shows that the flux tube has grown in horizontal extent and almost fills the computational box. We therefore also present the equivalent vertical temperature slice from simulation B1 which has a computational domain that is twice as large in the horizontal directions. It is evident also in this model that the rising flux sheet has pushed the transition region and lower corona aside and has lifted chromospheric plasma to heights of roughly $6 \mathrm{Mm}$. These cool, Lorentz force supported bubbles are long lived, and the simulation that has run the longest, B1 to $4500 \mathrm{~s}$, maintains an extended chromosphere for almost $2000 \mathrm{~s}$ and is still doing so at the end of the simulation. The gas in the extended chromospheric bubble is quite cool, down towards a uniform $2000 \mathrm{~K}$, and shows no sign of reheating at heights greater than $1 \mathrm{Mm}$. The big bubbles can also be seen in the synthetic Hinode Ca II H limb images as shown in figure 20. These synthetic limb observations, described in the figure, show that the chromosphere has expanded and cooled significantly as the flux tube rises through the chromosphere. Towards the end of the B1 simulation the emerging magnetic flux shows signs of having begun fragmentation as it reconnects with the previously present field. This looks to have interesting implications for transition region and coronal observables, but the process is not complete in any of our simulations and we will refrain from discussing this until the next paper in this series.

Further insight into this phenomena can be found by considering vertical slices of the B1 simulation at earlier times, as the flux tube bubble rises through the chromosphere, such as the slices of logarithmic temperature, vertical velocity and logarithmic pressure we show in figure 21. We find that a small, low $\beta$ region forms just above the photosphere, centrally placed over the (partly stalled) rising flux tube. As plasma $\beta$ falls below one, the bubble becomes buoyant and rises at roughly the sound speed, expanding rapidly both horizontally and vertically as it does so. The expansion causes the plasma to cool and the pressure in the bubble becomes much smaller than in the surrounding chromosphere. At some point in the chromosphere the bubble reaches a quasi-equilibrium with its surroundings and the continued expansion is halted, or at least slows considerably. 
The appearance of the extended chromospheric bubble and its associated magnetic field has large repercussions to chromospheric dynamics and energetics. This is abundantly clear as we consider figure 22 where we have plotted the variations of the temperature, velocity, and logarithmic pressure at height $1.1 \mathrm{Mm}$ as a function of time in the B1 simulation. The region where the bubble is found has very low temperatures and pressures, but also shows that the otherwise all pervasive chromospheric oscillations are excluded from the bubble. Acoustic waves seem to have difficulty entering this low $\beta$ region - and even when the bubble oscillates as the rest of the chromosphere at late times the temperature and pressure plots show that these oscillation are, at best, only weakly compressive. This expulsion process appears in every simulation we have run and is important from roughly $800 \mathrm{~km}$ above the photosphere and extends up to the transition region.

\section{Conclusions}

The emergence of magnetic flux tubes has been studied in order to understand not only the dynamics of flux emergence evolution, but also its effects on the solar atmosphere. One of the major questions we are considering is how flux tube emergence changes the energetics and the dynamics of the photosphere, chromosphere and corona. What contribution to atmospheric energetics does the emerging flux represent? Another set of important questions relates to the observational signatures of flux emergence throughout the atmosphere. This paper does not pretend to fully answer these questions, but is rather a first attempt to consider a framework for some of the problems and processes that arise as one follows the rise of a magnetic field up through the atmosphere.

Specifically, the aim of this work is to simulate a 3D box which contains a realistic convection zone and the atmospheric elements above; a photosphere and chromosphere with attendant non-grey radiative losses, a transition region and lower corona, including realistic optically thin cooling and Spitzer conductivity. The magnetic field injected at the lower boundary is either a horizontal flux tube with varying degrees of twist and magnetic field strengths, or a non-twisted flux sheet.

Considering first the region spanning the convection zone to photosphere, we find our results to agree with those reported by Cheung et al. (2007) as well as the observations of flux emergence of small-scale magnetic loops in the quiet-Sun internetwork studied by Centeno et al. (2007).

We also find that the time evolution of the magnetic flux tube as it rises into the atmospheric layers above the photosphere is similar in all five simulations we have studied. 
The flux tube or flux sheet crosses the different layers of the atmosphere at roughly the same time in each simulation as shown in table 2 .

The results shown in this paper focus on four different layers; the photosphere at height $10 \mathrm{~km}$, the region that produces reverse granulation at height $234 \mathrm{~km}$ and is represented by the bulk of the emission in the Hinode/SOT Ca II H-line, at height $458 \mathrm{~km}$ which is typical for the TRACE $160 \mathrm{~nm}$, and into the chromosphere proper at a height $906 \mathrm{~km}$ above the photosphere where short wavelength continuum emission at $130 \mathrm{~nm}$ and $109 \mathrm{~nm}$ are observable with SOHO/SUMER. Simulation A4 is the longest running simulation that contains a twisted flux tube. In this simulation the flux tube rises from the convection zone to the chromosphere at height around $1.1 \mathrm{Mm}$ in $3000 \mathrm{~s}$. The amount of magnetic flux that reaches each of these regions is strongly related to the twist parameter and also to the original magnetic field strength injected at the bottom boundary. We find that with greater twist and/or greater field strength, more flux crosses the photosphere into the chromosphere. The photosphere is the region where most of the flux is retained. The flux that eventually enters the lower chromosphere rises through the various heights we have recorded at less than $10 \mathrm{~km} \mathrm{~s}^{-1}$ and does not halt until reaching the transition region/lower corona.

As previously reported in the literature, we find that an emerging flux tube produces an expansion of the granulation cells in the photosphere. This expansion begins some $400 \mathrm{~s}$ before the flux tube itself reaches the photosphere. The simulations show that also the chromospheric plasma expands as the emerging flux tube rises. As in the photosphere, we find a delay between cell expansion and the arrival of magnetic flux in the lower layers of the chromosphere. As a result of the expansion the tube plasma is colder than its surroundings, and emission from the central parts of the flux tube dim successively at all heights of the atmosphere according to the schedule outlined in table 2. Such successive dimming has been observed by the SOT and EIS instruments aboard the Hinode spacecraft (Hansteen et al. 2007) for lines up to and including Fe XII $19.5 \mathrm{~nm}$ which is formed at a temperature of some 1.2 MK, but further observations are certainly required to search for confirmation or discrepancies with the models described here.

In addition to dimming of the central portion of the flux tube, the expansion produces other important effects: For instance, in the photosphere, the boundary layer of the rising flux tube is a region that is conducive in producing granular collapse.

We also find that chromospheric structure is substantially altered by the rise and expansion of the flux tube. The chromosphere is pushed upward by the magnetic field and cool chromospheric material is found at heights up to $6 \mathrm{Mm}$ above the photosphere in contrast to the 2-3 Mm extent of the pre-emergence chromosphere. In expanding, the transition region and corona above the flux tube are pushed aside by the chromospheric plasma. How- 
ever, the pre-existing coronal magnetic field and transition region and coronal emission are remarkably little changed at the times reached by our simulations. Only in simulation B1 do we see some initial signs of reconnection between the pre-existing coronal field and the rising bubble of emerging flux. This reconfiguration, with attendant changes in transition region and coronal emission, occurs some 30-40 minutes after the flux tube initially pierces the photosphere. Since the flux tube also expands dramatically in the horizontal direction, reaching widths almost equal to the simulation box for simulations A1-A4, discussion of transition region and coronal effects will be postponed to a later paper when simulations contained in a larger box are completed.

While the cooling process in the photosphere and at the lower chromospheric heights $(234,458 \mathrm{~km})$ is dominated by adiabatic cooling, we find that at higher layers where $\beta<1$ other processes also come into play. The acoustic waves that are generated as a result of convective overshoot and/or granular dynamics and that permeate the non-magnetic chromosphere are expelled from the rising cooling flux tube. Thus, the temperature variations that are due to compressive shock waves propagating up through the chromosphere are absent in the plasma rising with the flux tube which maintains a much lower temperature than its surroundings for the duration of our simulations. Towards the end of the simulations the magnetic bubble does begin to oscillate with the same vertical velocity as the rest of the atmosphere, but the modes penetrating the bubble are still non-compressive at the time of the completion of the simulation and the temperature remains fairly constant.

Another interesting effect is the increase of magnetic field strength in small regions or "points" in the atmosphere near the edges of the rising flux tube. For example, photospheric bright points are formed in the intergranular lanes edging the flux tube after the flux tube has passed through the photosphere. These are regions where the magnetic field is concentrated and the field reaches strengths greater than $10^{3} \mathrm{G}$. Such bright points have been extensively observed in the G-band and other spectral windows previously and theoretical and numerical work have explained their appearance: The high intensity of bright points is explained by the high magnetic pressure causing a low gas density. The resulting low opacity means that intensity is formed in regions below, where the gas temperature is higher. However, we also find bright points in the overlying regions formed as a result of flux emergence. In the case of the higher lying regions the bright points are regions of concentrated high magnetic field strength, high up- or down-flowing velocity, high velocity convergence and high vorticity. They are bright in the various spectral bands describing these heights as a result of high gas temperature due to the compression of the fluid in these regions.

There are several interesting and important effects that we have not reported extensively in this paper, amongst these are the effects of joule heating and transition region and coronal 
diagnostics of flux emergence. This is mainly due to the finding that the flux emergence process is a quite slow one: it takes on the order of one hour solar time from the time the magnetic field pierces the photosphere until it reaches and begins to recombine with the preexisting coronal field. We are currently undertaking simulations that have run long enough and that have a large enough computational box to study these phenomena.

\section{Acknowledgements}

This research was supported by the Research Council of Norway through grant 170935/V30 and through grants of computing time from the Programme for Supercomputing and by a Marie Curie Early Stage Research Training Fellowship of the European Community's Sixth Framework Programme under contract number MESTCT-2005-020395. Financial support by the European Commission through the SOLAIRE Network (MTRN-CT-2006035484) is gratefully acknowledged. To analyze the data we have used IDL and Vapor (www.vapor.ucar.edu). 


\section{REFERENCES}

Abbett W. P., 2007, ApJ, 665, 1469

Acheson D. J., 1979, Sol. Phys., 62, 23

Archontis A., Moreno-Insertis F., Galsgaard K., 2004, A\&A, 426, 1047

Caligari P., Moreno-Insertis F., Schussler M., 1995, ApJ, 441, 886

Carlsson M., Hansteen V. H., De Pontieu B., et al., 2007, ArXiv e-prints, 709

Carlsson M., Stein R. F., 1992, ApJ, 397, L59

Carlsson M., Stein R. F., 1994, in Carlsson M. (ed.), Chromospheric Dynamics, p. 47

Carlsson M., Stein R. F., 1995, ApJ, 440, L29

Carlsson M., Stein R. F., 1997, ApJ, 481, 500

Carlsson M., Stein R. F., 2002, ApJ, 572, 626

Carlsson M., Stein R. F., Nordlund Å., Scharmer G. B., 2004, ApJ, 610, L137

Centeno R., Socas-Navarro H., Lites B., et al., 2007, ApJ, 666, L137

Cheung M. C. M., Moreno-Insertis F., 2006, A\&A, 451, 303

Cheung M. C. M., Schüssler M., Moreno-Insertis F., 2007, A\&A, 467, 703

Dorch S. B. F., Nordlund A., 1998, A\&A, 338, 329

Emonet T., Moreno-Insertis F., Rast M. P., 2001, ApJ, 549, 1212

Evans J. W., Catalano C. P., 1972, Sol. Phys., 27, 299

Fan Y., 2001, ApJ, 554, L111

Fan Y., Fischer G. H., DeLuca E. E., 1993, Sol Phys, 405, 390

Fan Y., Zweibel E. G., Linton M. G., Fischer G. H., 1998, ApJ, 505, L59

Gudiksen B. V., Nordlund Å., 2004, An Ab Initio Approach to the Solar Coronal Heating Problem, in IAU Symposium, Vol. 219, Dupree A. K., Benz A. O. (eds.), Stars as Suns : Activity, Evolution and Planets, p. 488

Gudiksen B. V., Nordlund A., 2005, ApJ, 618, 1020, 
Hansteen V. H., 2004, Initial simulations spanning the upper convection zone to the corona, in IAU Symposium, Vol. 223, Stepanov A. V., Benevolenskaya E. E., Kosovichev A. G. (eds.), Multi-Wavelength Investigations of Solar Activity, p. 385

Hansteen V. H., Carlsson M., Gudiksen B., 2007, 3D Numerical Models of the Chromosphere, Transition Region, and Corona, in Astronomical Society of the Pacific Conference Series, Vol. 368, Heinzel P., Dorotovič I., Rutten R. J. (eds.), The Physics of Chromospheric Plasmas, p. 107

Hansteen V. H., De Pontieu B., Carlsson M., et al., 2007, ArXiv e-prints, 711

Hyman J., Vichnevtsky R., Stepleman R., 1979, Adv. in Comp. Meth, PDE's-III, 313

Judge P. G., Meisner R. W., 1994, The 'HAO spectral diagnostics package' (HAOS-Diaper), in ESA Special Publication, Vol. 373, Hunt J. J. (ed.), Solar Dynamic Phenomena and Solar Wind Consequences, the Third SOHO Workshop, p. 67

Leenaarts J., Wedemeyer-Böhm S., 2005, A\&A, 431, 687

Linton M. G., Longcope D. W., Fisher G. H., 1996, ApJ, 469, 954

Lites B. W., V. M., Seagraves P., Skunanick A., 1995, ApJ, 446, 877

Longcope D. W., Fischer G. H., Arendt S., 1996, ApJ, 464, 999

Mackay D. H., Galsgaard K., 2001, Sol. Phys., 198, 289

Magara T., 2004, ApJ, 605, 480

Magara T., 2006, ApJ, 653, 1499

Magara T., Longcope D. W., 2001, ApJ, 559, L55

Manchester W., IV, Gombosi T., DeZeeuw D., Fan Y., 2004, ApJ, 610, 588

Matsumoto R., Tajima T., Chou W., Okubo A., Shibata K., 1998, ApJ, 493, L43

Moreno-Insertis F., 1986, A\&A, 166, 291

Moreno-Insertis F., Emonet T., 1996, ApJ, 472, L53

Nordlund A., 1982, Aap, 107, 1

Rutten R. J., de Wijn A. G., Sütterlin P., 2004, A\&A, 416, 333 
Rutten R. J., Krijger J. M., 2003, A\&A, 407, 735

Schüssler M., 1979, A\&A, 71, 79

Shelyag S., Schüssler M., Solanki S. K., Berdyugina S. V., Vögler A., 2004, A\&A, 427, 335

Shibata K., Tajima T., Steinolfson R. S., Matsumoto R., 1989, ApJ, 345, 584

Skartlien R., 2000, ApJ, 536, 465

Skartlien R., Stein R. F., Nordlund Å., 2000, ApJ, 541, 468

Stein R. F., Bercik D., Nordlund A., 2003, Solar Surface Magneto-Convection, in Astronomical Society of the Pacific Conference Series, Vol. 286, Pevtsov A. A., Uitenbroek H. (eds.), Current Theoretical Models and Future High Resolution Solar Observations: Preparing for ATST, p. 121

Stein R. F., Nordlund A., 2002, Solar Surface Magneto-Convection and Dynamo Action, in ESA Special Publication, Vol. 505, Sawaya-Lacoste H. (ed.), SOLMAG 2002. Proceedings of the Magnetic Coupling of the Solar Atmosphere Euroconference, p. 83

Stein R. F., Nordlund A., 2006, ApJ, 642, 1246

Suemoto Z., Hiei E., Nakagomi Y., 1987, Sol. Phys., 112, 59

Suemoto Z., Hiei E., Nakagomi Y., 1990, Sol. Phys., 127, 11

Vögler A., Shelyag S., Schüssler M., et al., 2005, A\&A, 429, 335

Wedemeyer S., Freytag B., Steffen M., Ludwig H.-G., Holweger H., 2004, A\&A, 414, 1121 

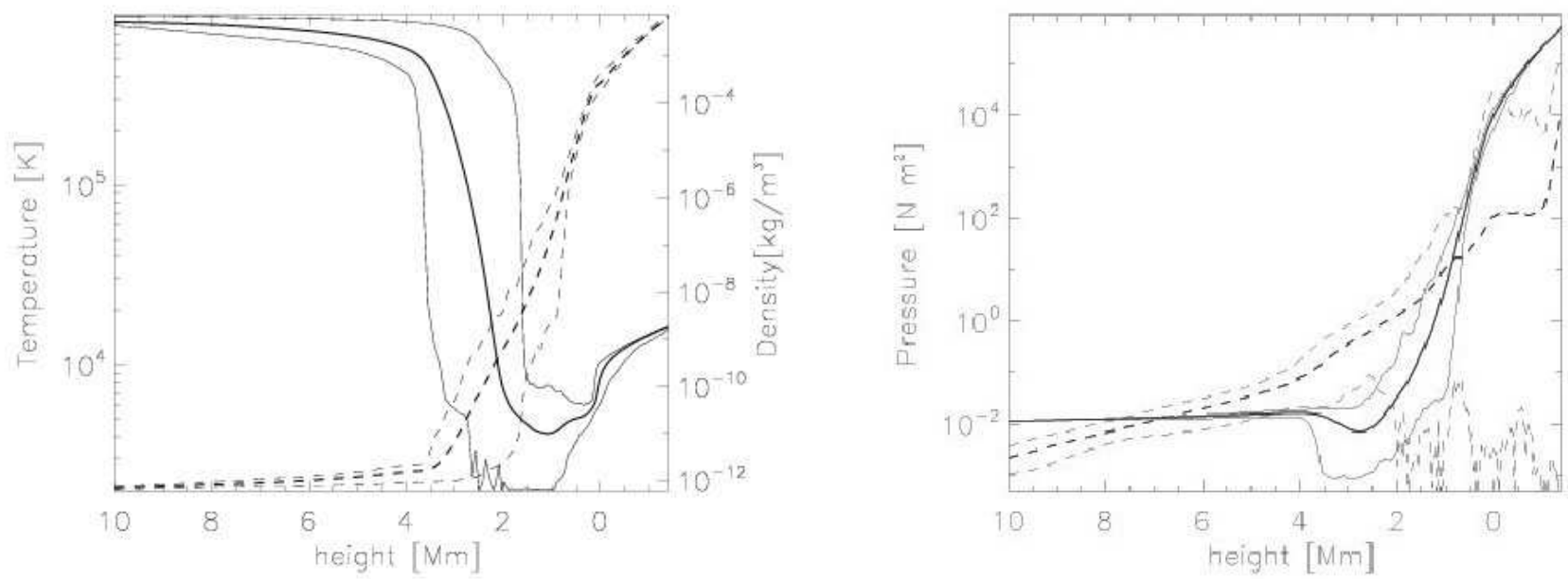

Fig. 1. - Left panel: Temperatures (solid) and densities (dashed). Right panel: Gas (solid) and magnetic (dashed) pressures. All as a function of height in the initial model. Minimum and maximum values are shown in grey, average values are shown in black. The photosphere is situated at $z \approx 0 \mathrm{Mm}$. The models extent in height is from -1.4 to $14 \mathrm{Mm}$ (top $4 \mathrm{Mm}$ not shown here). 


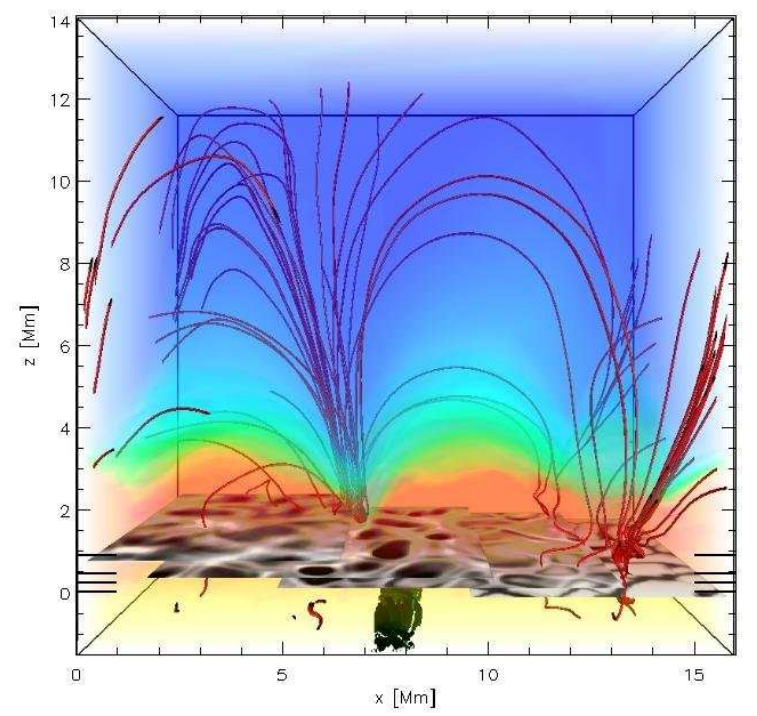

Fig. 2.- The computational box from the convection zone to the corona. The color table is the temperature. The magnetic flux tube coming from the bottom boundary (green lines). The initial (weak) background magnetic field lines (red lines). The $z$ axis runs from the corona ( $z=14 \mathrm{Mm}$ top boundary) to the convection zone ( $z=-1.4 \mathrm{Mm}$ bottom boundary). We center our attention to 4 different heights shown in the figure as partial planes with the temperature (grey-scale coded); at heights $z=10 \mathrm{~km}$ (photosphere), $z=234 \mathrm{~km}$ (reverse granulation), $z=458 \mathrm{~km}$ (photosphere-chromosphere) and $z=906 \mathrm{~km}$ (mid-chromosphere). The black lines at the left and right side show the heights of the four layers. 

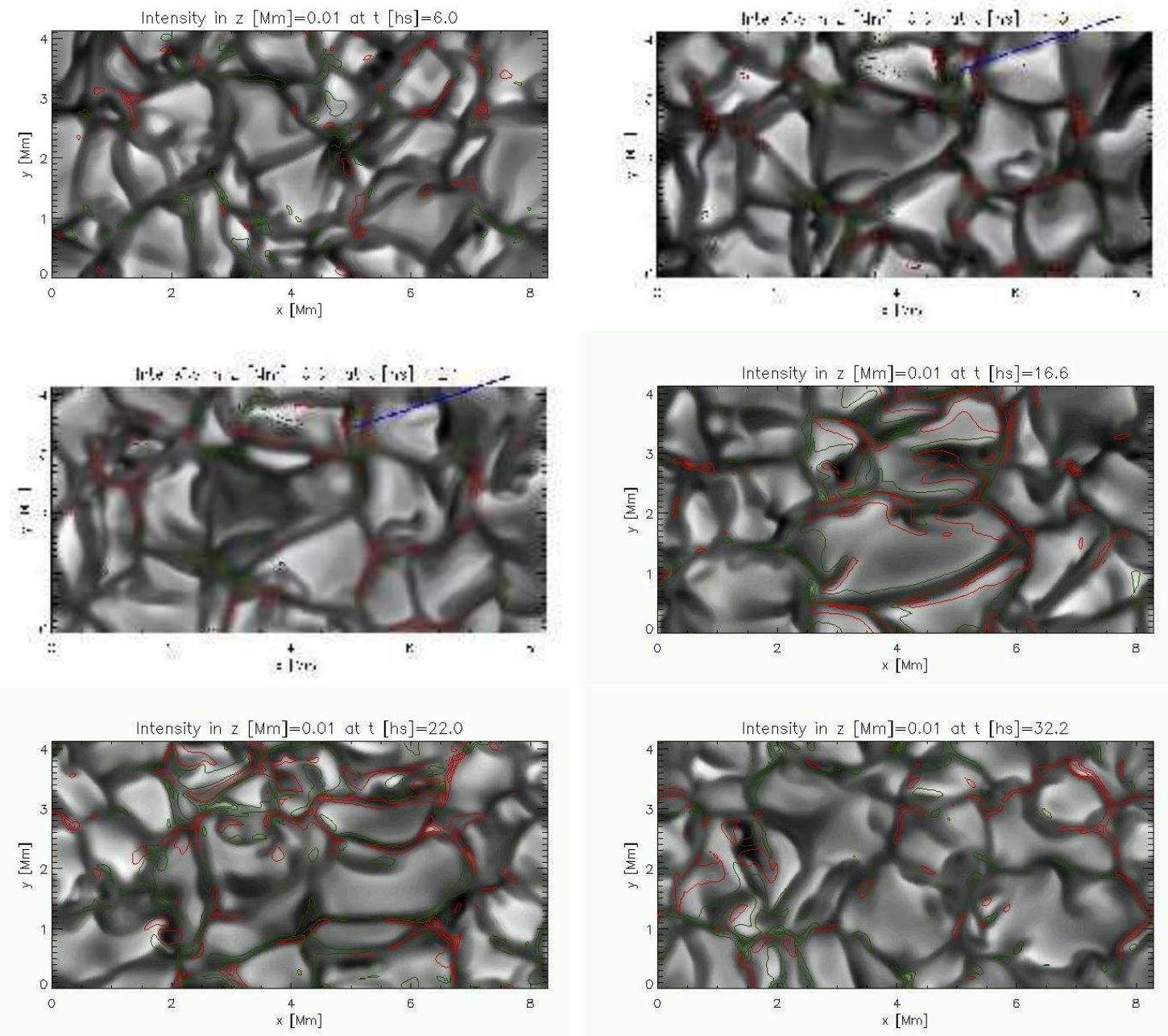

Fig. 3.- The continuum intensity shown at times $600 \mathrm{~s}$, before the flux tube goes through the photosphere, at time $1100 \mathrm{~s}$, when the flux tube is close to the photosphere, at time $1210 \mathrm{~s}$, when the big cells are cooling by expansion, at time $1660 \mathrm{~s}$, when the tube is crossing the photosphere, at time $2200 \mathrm{~s}$, when the structure of the cells appears to have returned back to normal and at time $3220 \mathrm{~s}$, from the left to right and top to bottom respectively. The red contours correspond to positive $B_{z}$ and green contours to negative $B_{z}$ at $z=10 \mathrm{~km}$. An example of a collapsed granule is marked with a blue line. 

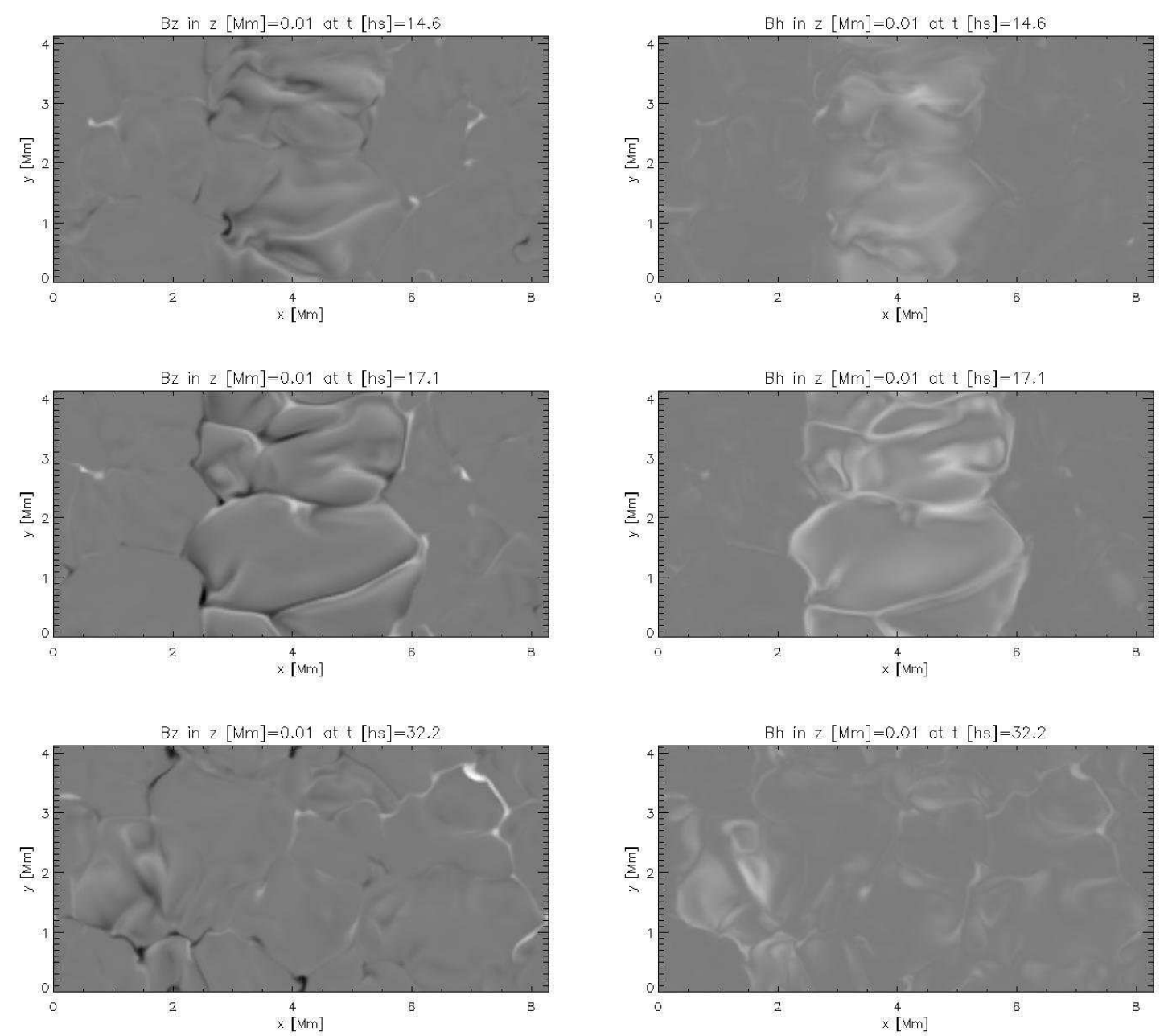

Fig. 4.- $B_{z}$ (longitudinal "magnetograms") in the photosphere, at $z=10 \mathrm{~km}$, shown with grey-scale intensity at three different times in the simulation in the left panels. $B_{h}$ (horizontal magnetic field) in the photosphere at the same height and same instants shown in the right panels. The grey-scale goes from $-1111.5 \mathrm{G}$ to $1376.3 \mathrm{G}$. The times chosen are at time $1460 \mathrm{~s}$, when the tube just has started to cross the photosphere (top panels); at time $1710 \mathrm{~s}$, as the tube is well into the photosphere (middle panels); and at time $3220 \mathrm{~s}$ (bottom panels). 

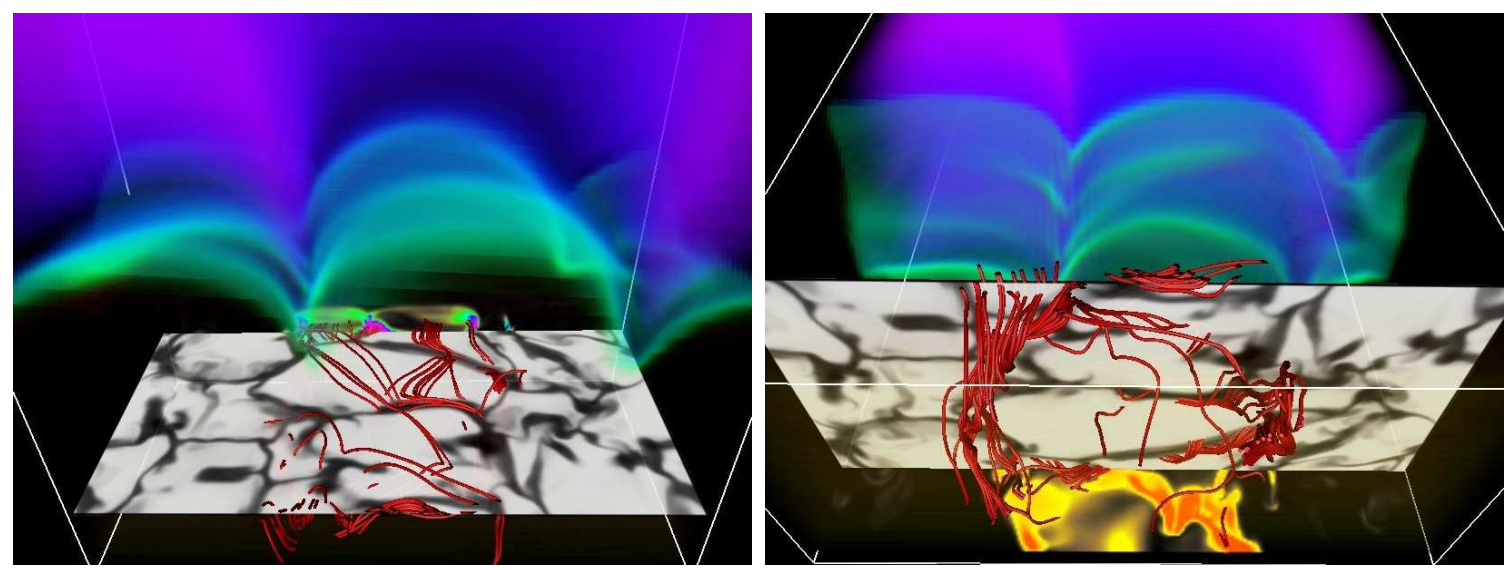

Fig. 5. - 3D image of the simulation at time $t=1700 \mathrm{~s}$. Temperature at the photosphere shown with grey-scale intensity, the red lines are the magnetic field lines of the tube, temperatures in the corona shown with green-violet scale color (green is the transition region) and with orange-scale is the magnetic field strength. View from above (left panel) and from below (right panel). 


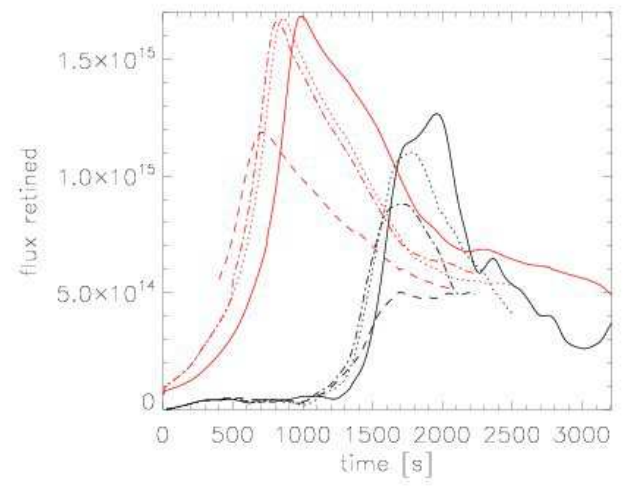

Fig. 6.- Mean magnetic flux per unit area perpendicular to the tube as function of time. Region confined between the $z=10 \mathrm{~km}$ and $z=254 \mathrm{~km}$ in black color and below the photosphere in red color for the runs A1 (dash-dot line), A2 (dashed line), A3 (dotted line) and A4 (solid line). 

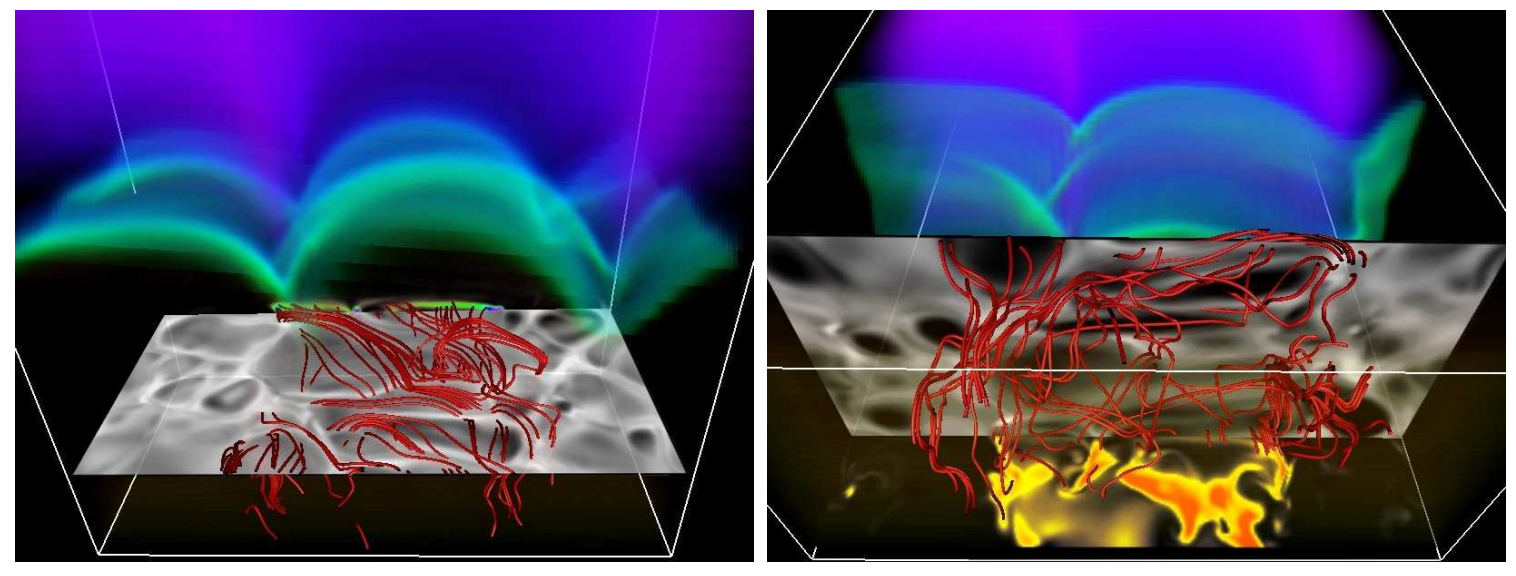

Fig. 7. - 3D image of the simulation at time $t=1900 \mathrm{~s}$. Temperature at the height $(z=234 \mathrm{~km})$ shown with grey-scale intensity. The rest of the color scale is the same as in figure 5. View from above (left panel) and from below (right panel). 

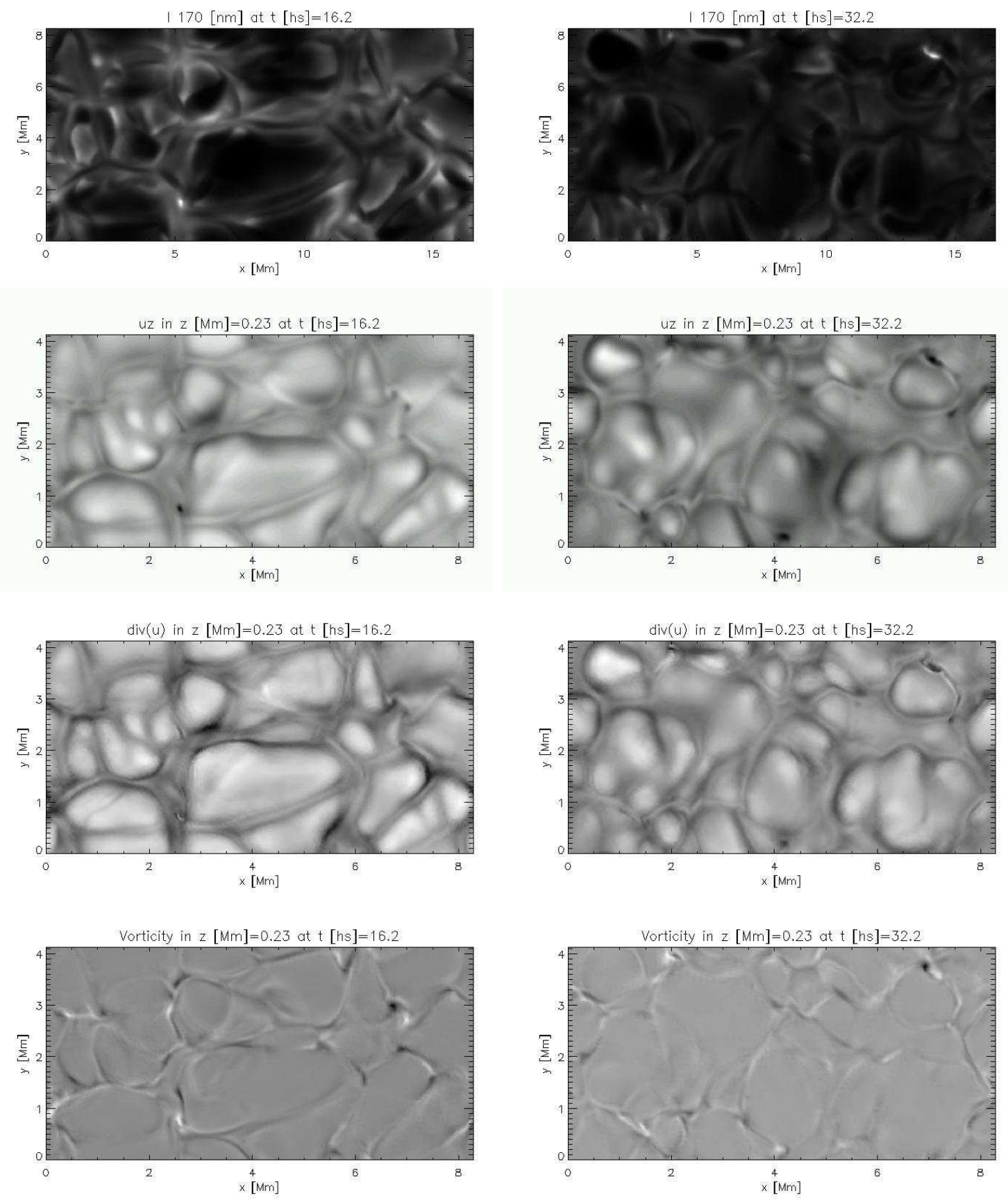

Fig. 8.- Continuum intensity at $170 \mathrm{~nm}$ (top row), vertical velocity (second row), divergence of the velocity (third row) and the vertical vorticity (bottom row) at $z=234 \mathrm{~km}$ at time $1620 \mathrm{~s}$ (left column) and $3220 \mathrm{~s}$ (right column). 

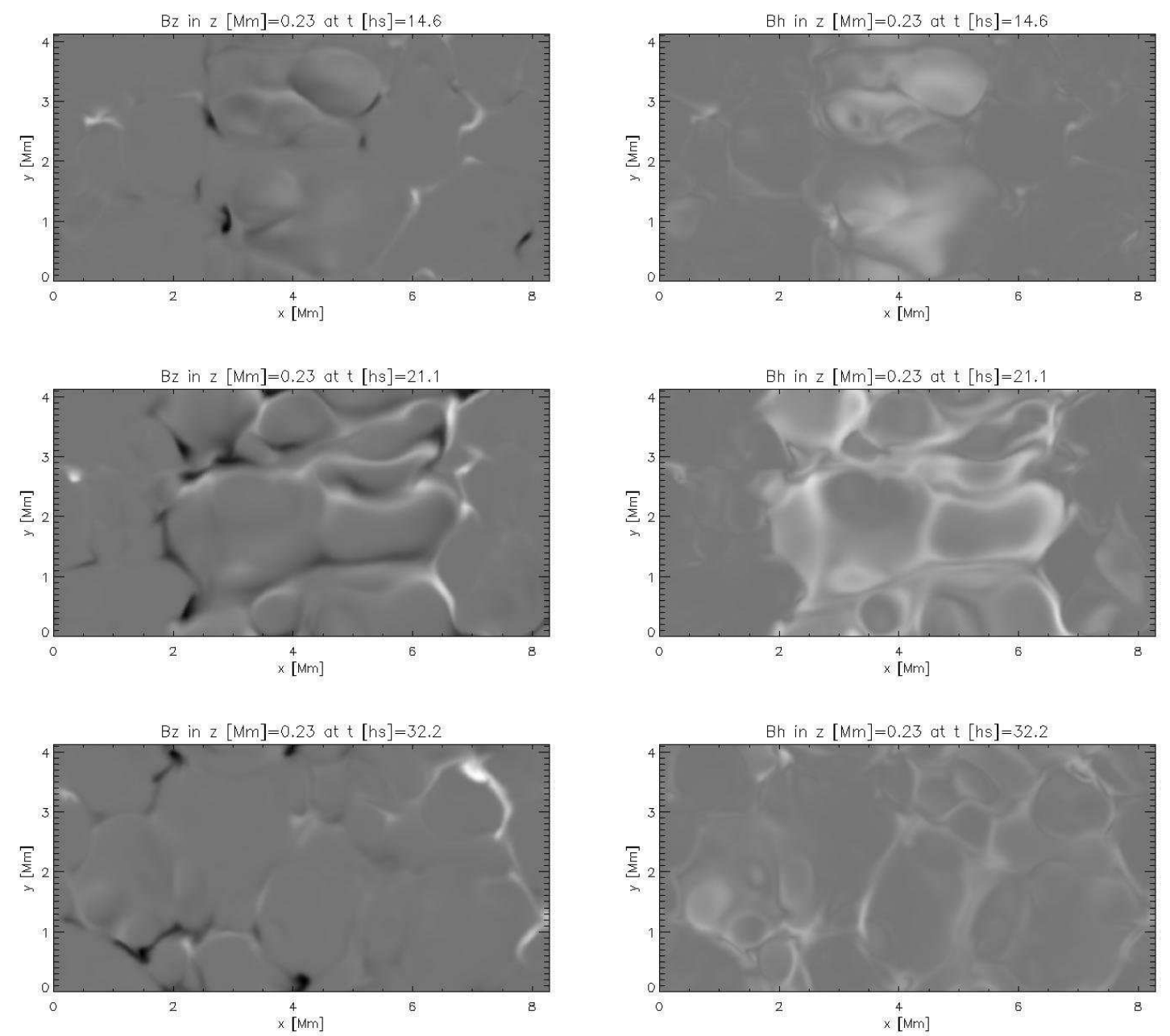

Fig. 9. $-B_{z}$ at $z=234 \mathrm{~km}$ (left panels) and $B_{h}$ (right panels). The grey-scale goes from $-560.3 \mathrm{G}$ to $659.5 \mathrm{G}$. The field is shown at times $1460 \mathrm{~s}$ (top panels) when the tube start to cross the reverse granulation layer, at time $2110 \mathrm{~s}$ when the tube is crossing the reverse granulation (middle panels) and at time $3220 \mathrm{~s}$ when some rest of the flux returns to the reverse granulation (bottom panels) 


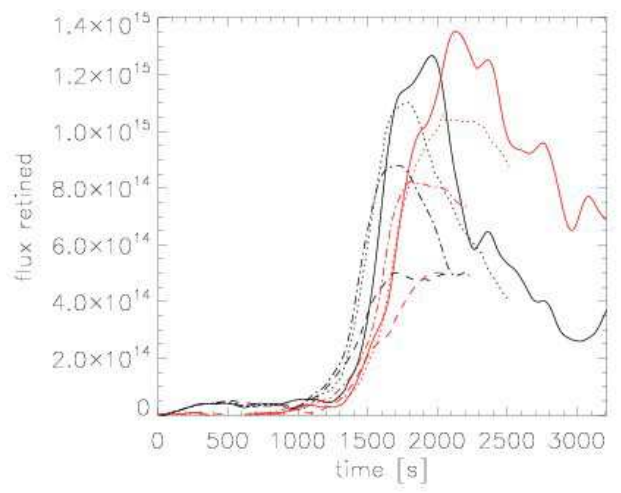

Fig. 10. - Variation in time of the mean magnetic flux by area as defined in equation 15 from the photosphere $(z=10 \mathrm{~km})$ to the height $z=234 \mathrm{~km}$ with black color and from the height $z=234 \mathrm{~km}$ to the height $z=458 \mathrm{~km}$ with red color for the runs A1 (dash-dot line), A2 (dashed line), A3 (dotted line) and A4 (solid line). 

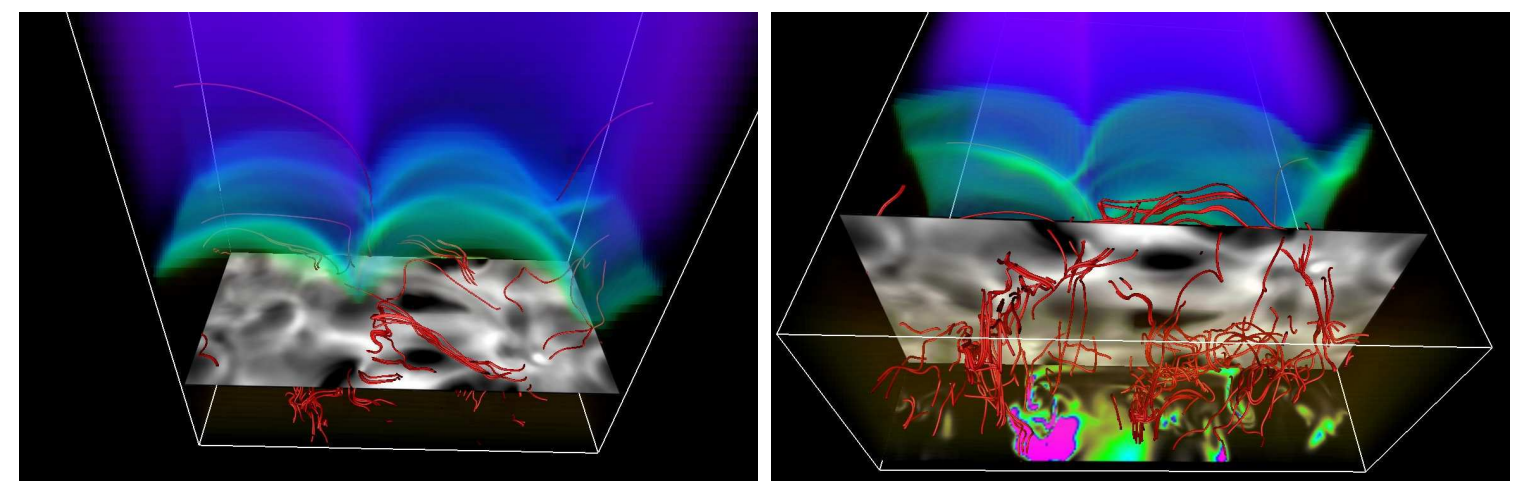

Fig. 11. - 3D image of the simulation at time $t=1980 \mathrm{~s}$. Temperature at $z=458 \mathrm{~km}$ shown with grey-scale intensity. The rest of the color scale is the same as in figure 5. View from above (left panel) and from below (right panel). 

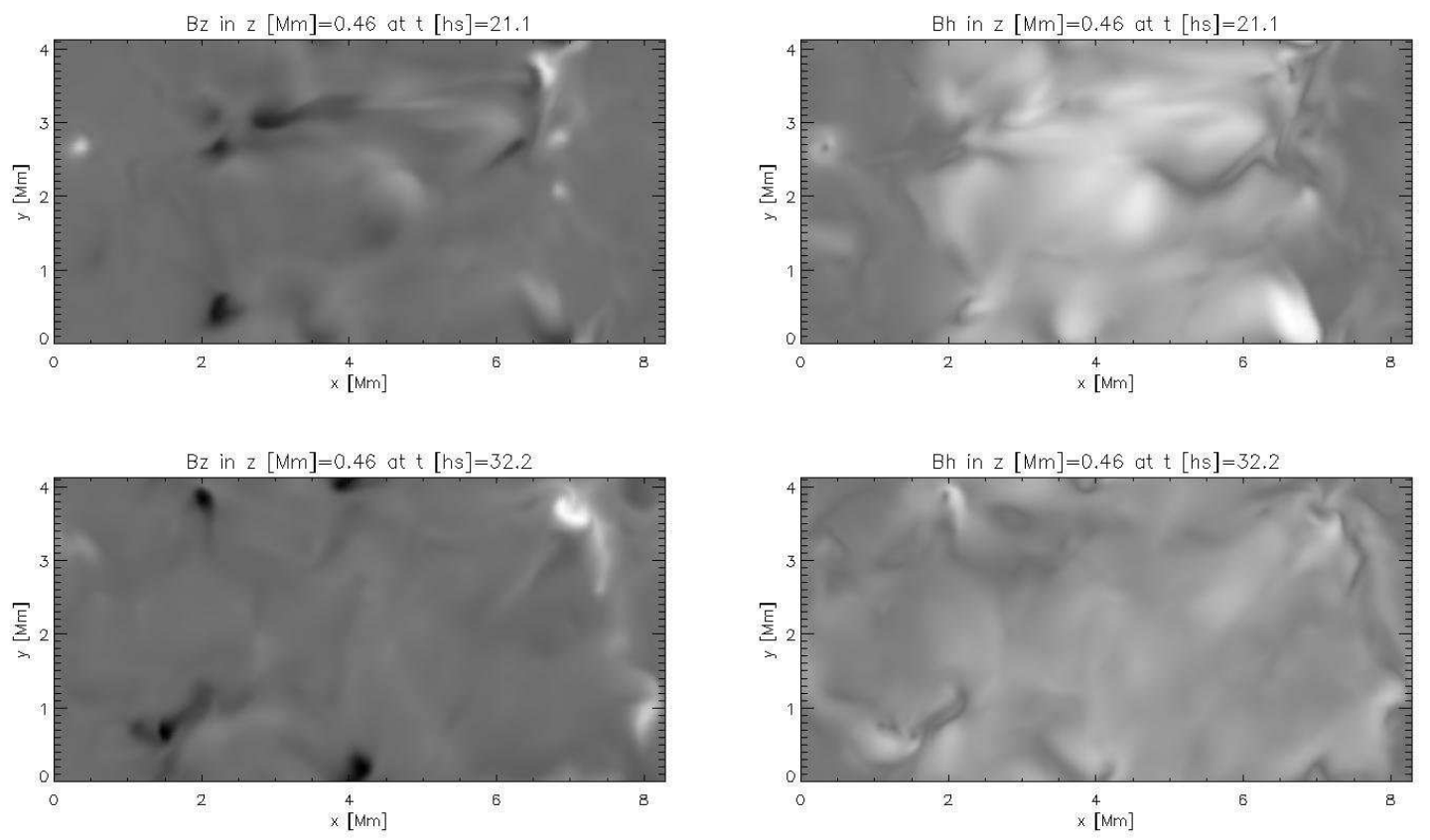

Fig. 12.- $B_{z}$ at $z=458 \mathrm{~km}$ (left panels) and $B_{h}$ (right panels) at times $2110 \mathrm{~s}$ when the tube is crossing the layer $458 \mathrm{~km}$ height (top panels) and at time $3220 \mathrm{~s}$ (bottom panels). The grey-scale range is $[-217,284] \mathrm{G}$ for all panels. 

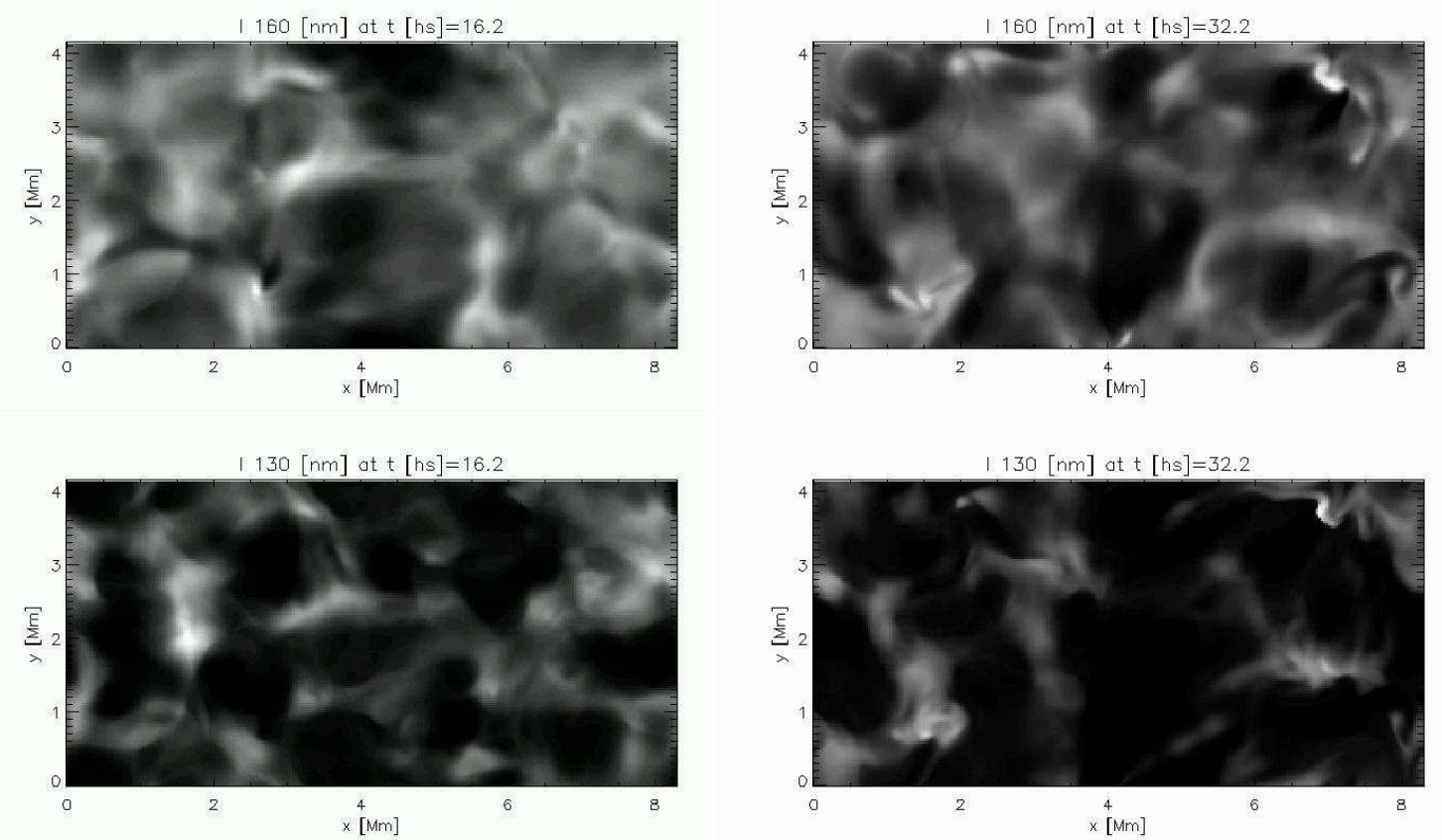

Fig. 13.- Continuum intensity to the power of 0.4 (equivalent to a gamma of 0.4 for an image) to better bring out the structure, at $160 \mathrm{~nm}$ (top row) and $130 \mathrm{~nm}$ (bottom row) at time $1620 \mathrm{~s}$ when the tube starts to enter the chromosphere (left column) and at $3220 \mathrm{~s}$ when the tube has crossed the height $z=458 \mathrm{~km}$ (right column) 

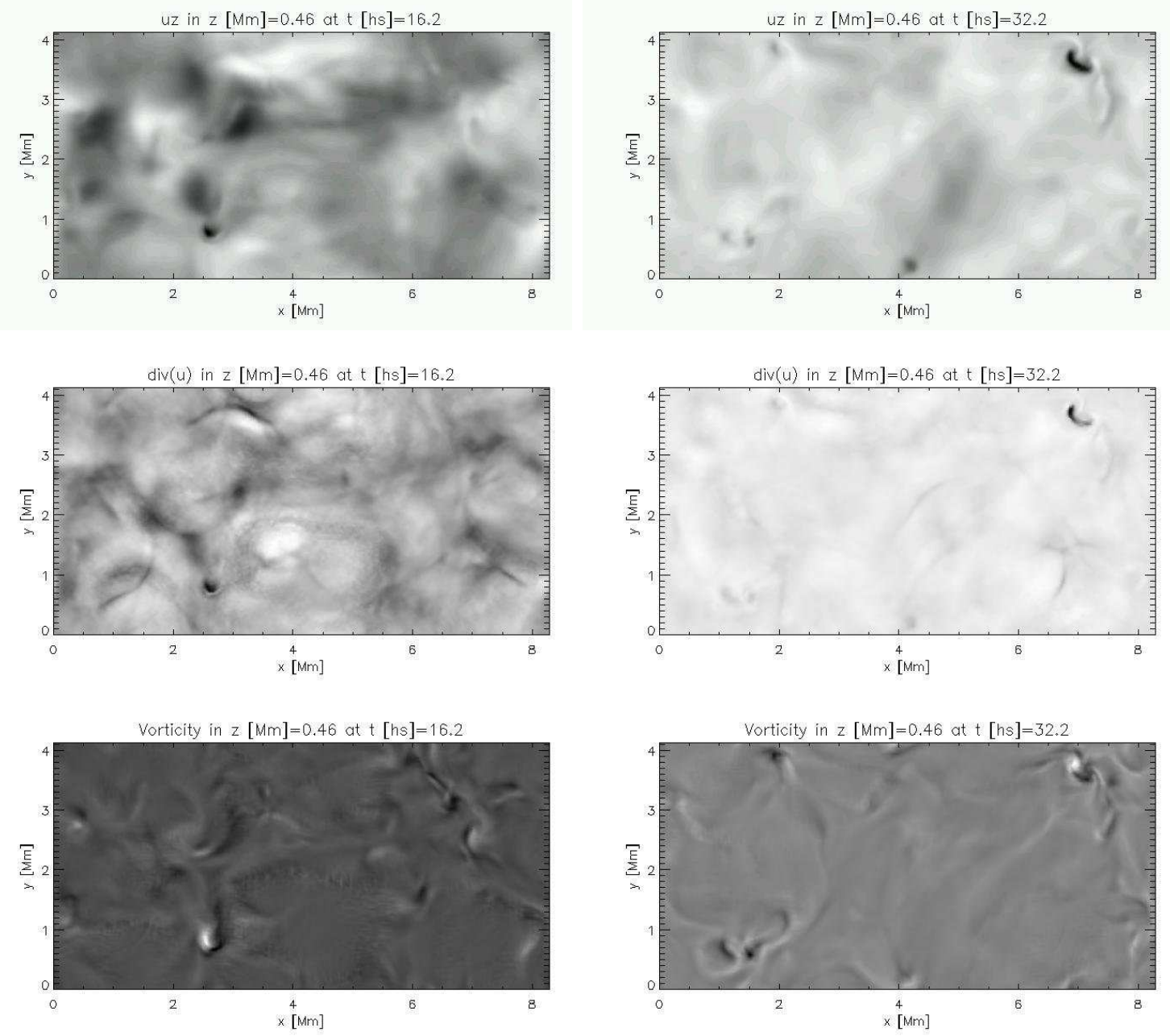

Fig. 14. - Vertical velocity (top row), divergence of the velocity (middle row) and the vertical vorticity (bottom row) at $z=458 \mathrm{~km}$ at time $1620 \mathrm{~s}$, when the flux tube is close to the layer at height $z=458 \mathrm{~km}$ (left column), and at time $3220 \mathrm{~s}$, when the tube has crossed the layer $458 \mathrm{~km}$ height (right column). 


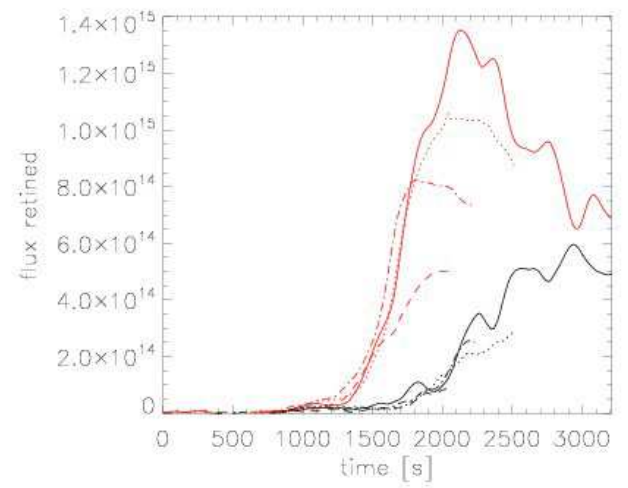

Fig. 15. - Mean magnetic flux per unit area perpendicular to the tube as function of time. Region confined between $z=234 \mathrm{~km}$ to $z=458 \mathrm{~km}$ in red color and from $z=458 \mathrm{~km}$ to $z=901 \mathrm{~km}$ in black color for the runs A1 (dash-dot line), A2 (dashed line), A3 (dotted line) and A4 (solid line). 

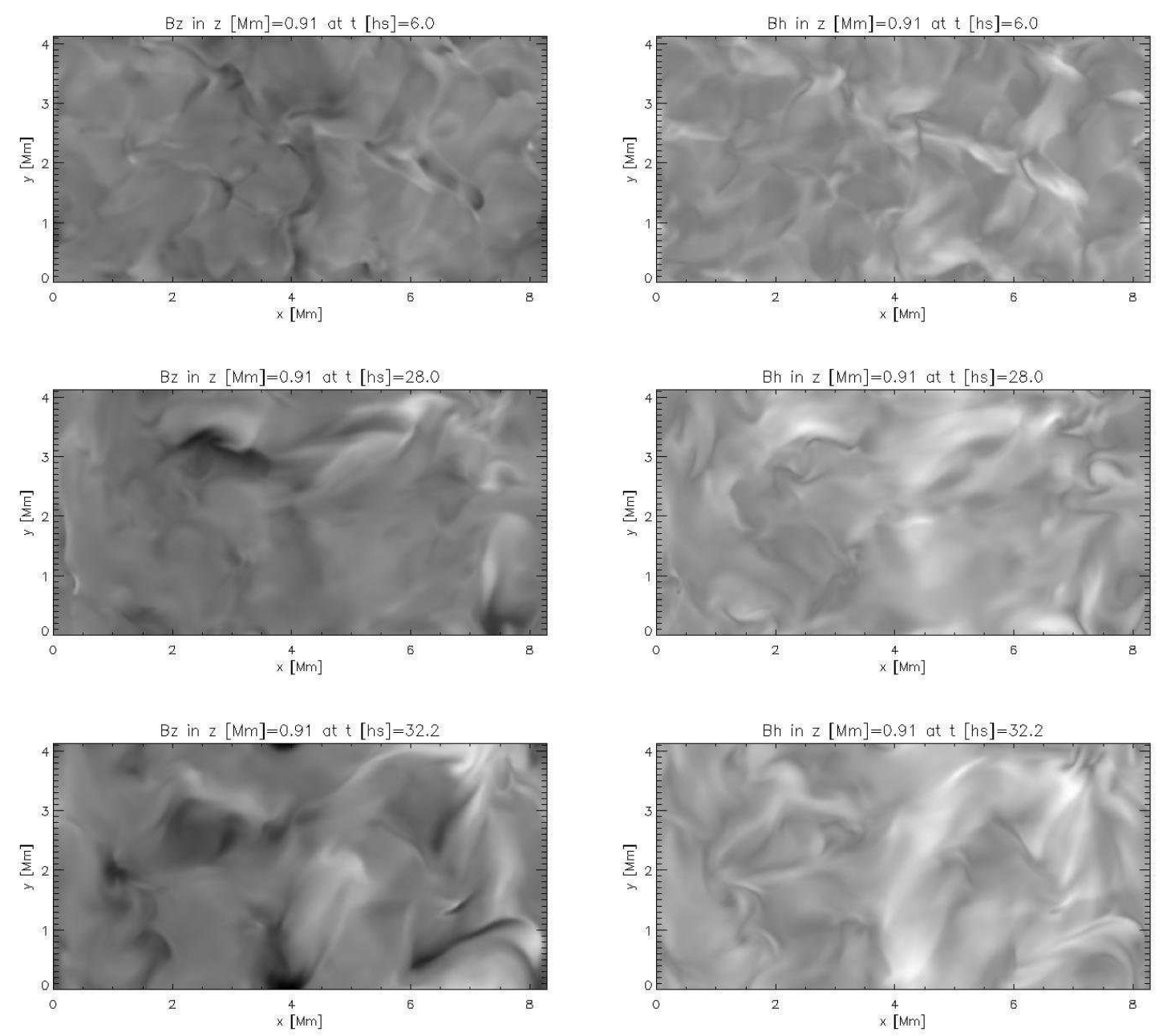

Fig. 16. - $B_{z}$ at $z=906 \mathrm{~km}$ (left panels), and $B_{h}$ (right panels) at times $600 \mathrm{~s}$, before the flux tube starts to cross the chromosphere (top panels), at time $2800 \mathrm{~s}$ when the tube is crossing the photosphere (middle panels) and at time $3220 \mathrm{~s}$ (bottom panels). The grey-scale goes from $-60.46 \mathrm{G}$ to $65.91 \mathrm{G}$ 

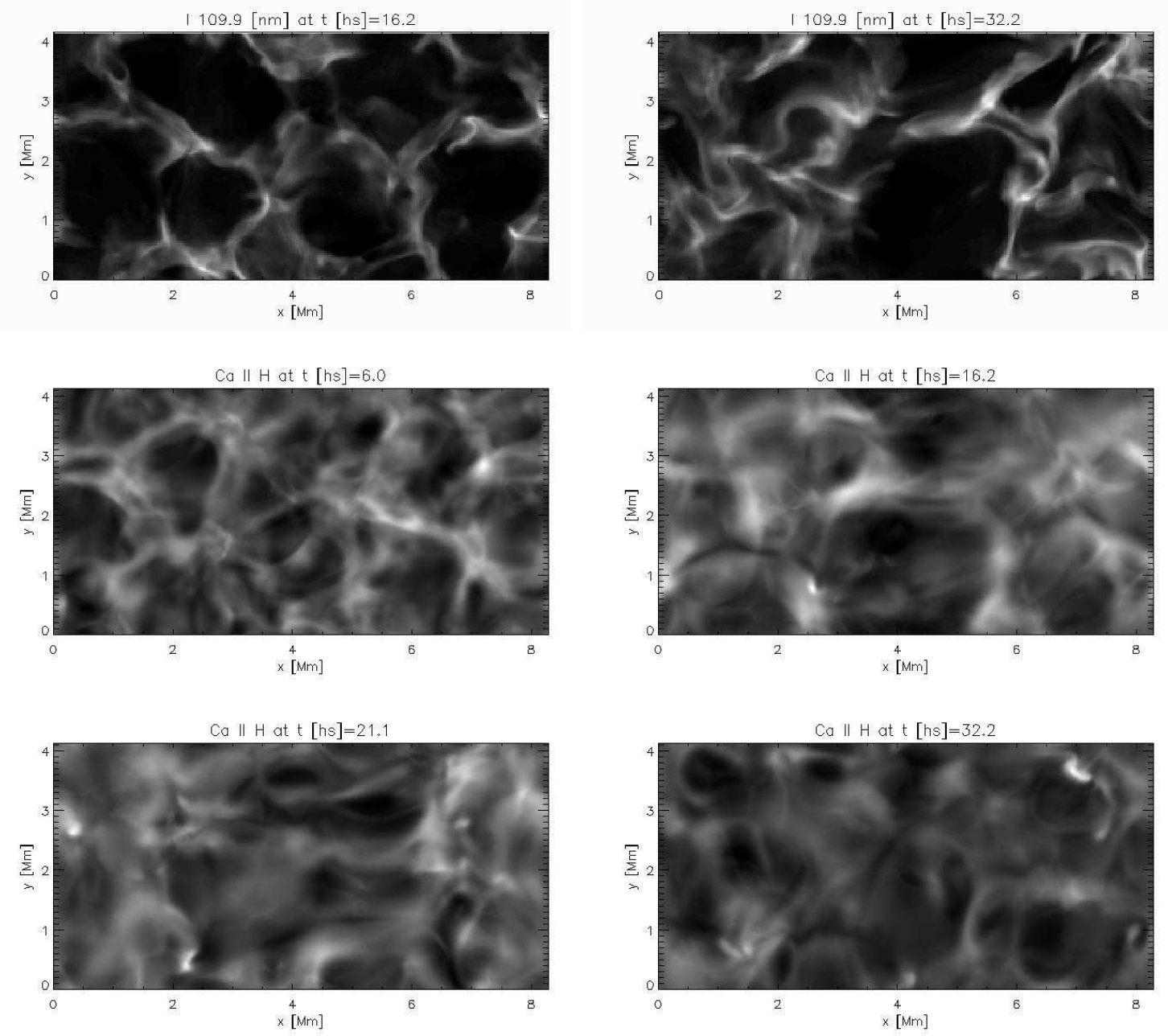

Fig. 17.- Continuum intensity of the $\mathrm{C}$ I to the power of 0.4 (equivalent to a gamma of 0.4 for an image) to better bring out the structure at $109.9 \mathrm{~nm}$ at time $1620 \mathrm{~s}$ (top left) and at time $3220 \mathrm{~s}$ (top right). The synthetic Ca II H line intensity at time $600 \mathrm{~s}$, before the flux tube goes through the chromosphere, at time $1620 \mathrm{~s}$ when the tube starts to cross, at time $2110 \mathrm{~s}$ as the tube is crossing, and at $3220 \mathrm{~s}$ when the tube has crossed the chromosphere, from the left to the right and top to bottom respectively. 
$u z$ in $z[M m]=0.91$ at $t[h s]=6.0$
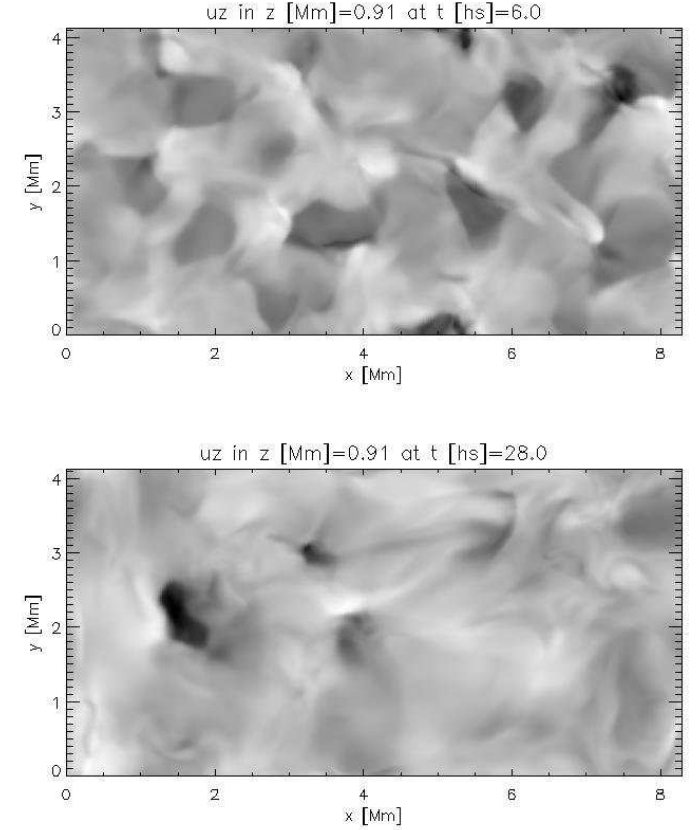
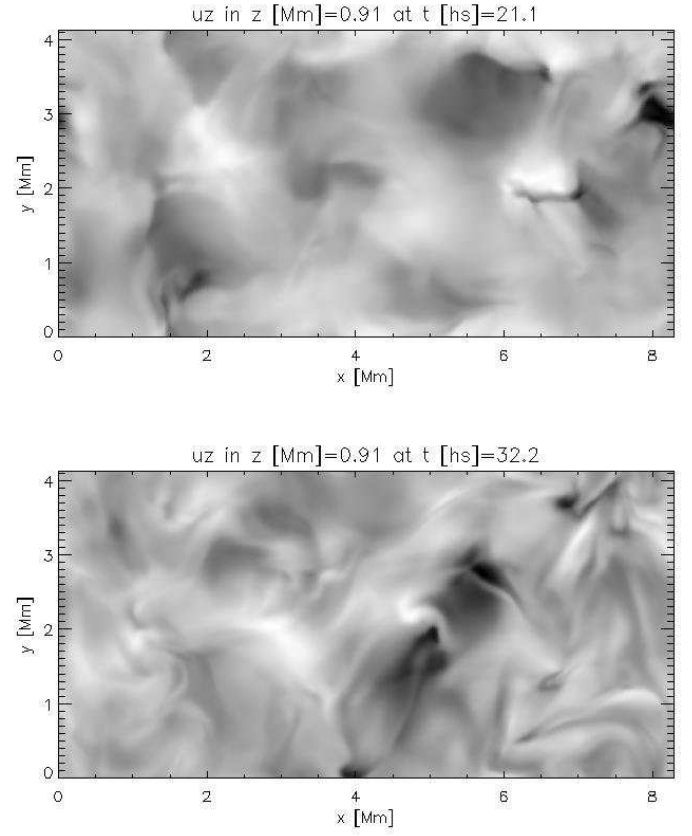

Fig. 18. - Vertical velocity at $z=906 \mathrm{~km}$ at times $600 \mathrm{~s}$, before the flux tube goes through the chromosphere (top left), at time $2110 \mathrm{~s}$, when the flux tube is closed to the chromosphere (top right), at time $2800 \mathrm{~s}$, when the big cells are cooling through expansion (bottom left), and at time $3220 \mathrm{~s}$, after the tube has passed through the chromosphere (bottom right). 

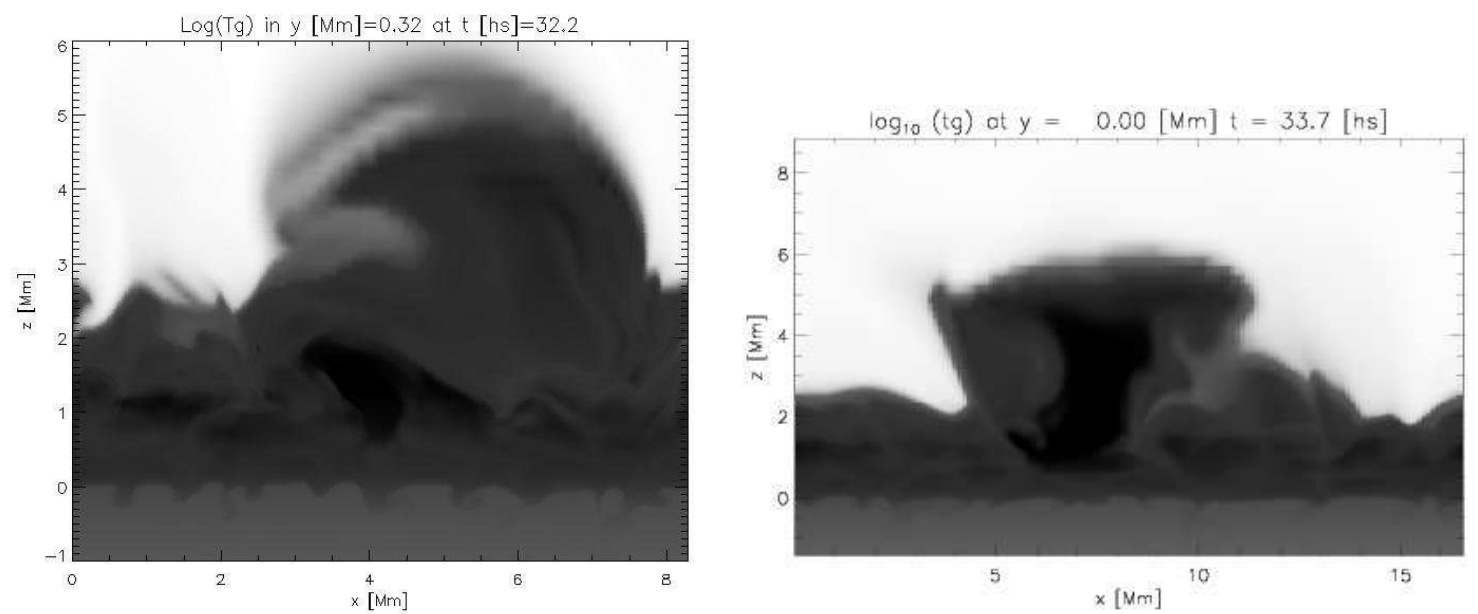

Fig. 19. - Temperature shown with grey-scale in a vertical plane at $3220 \mathrm{~s}$ for the simulations A4 (left) and for the simulation B1 at time $3370 \mathrm{~s}$ (right). Note the different scales. 

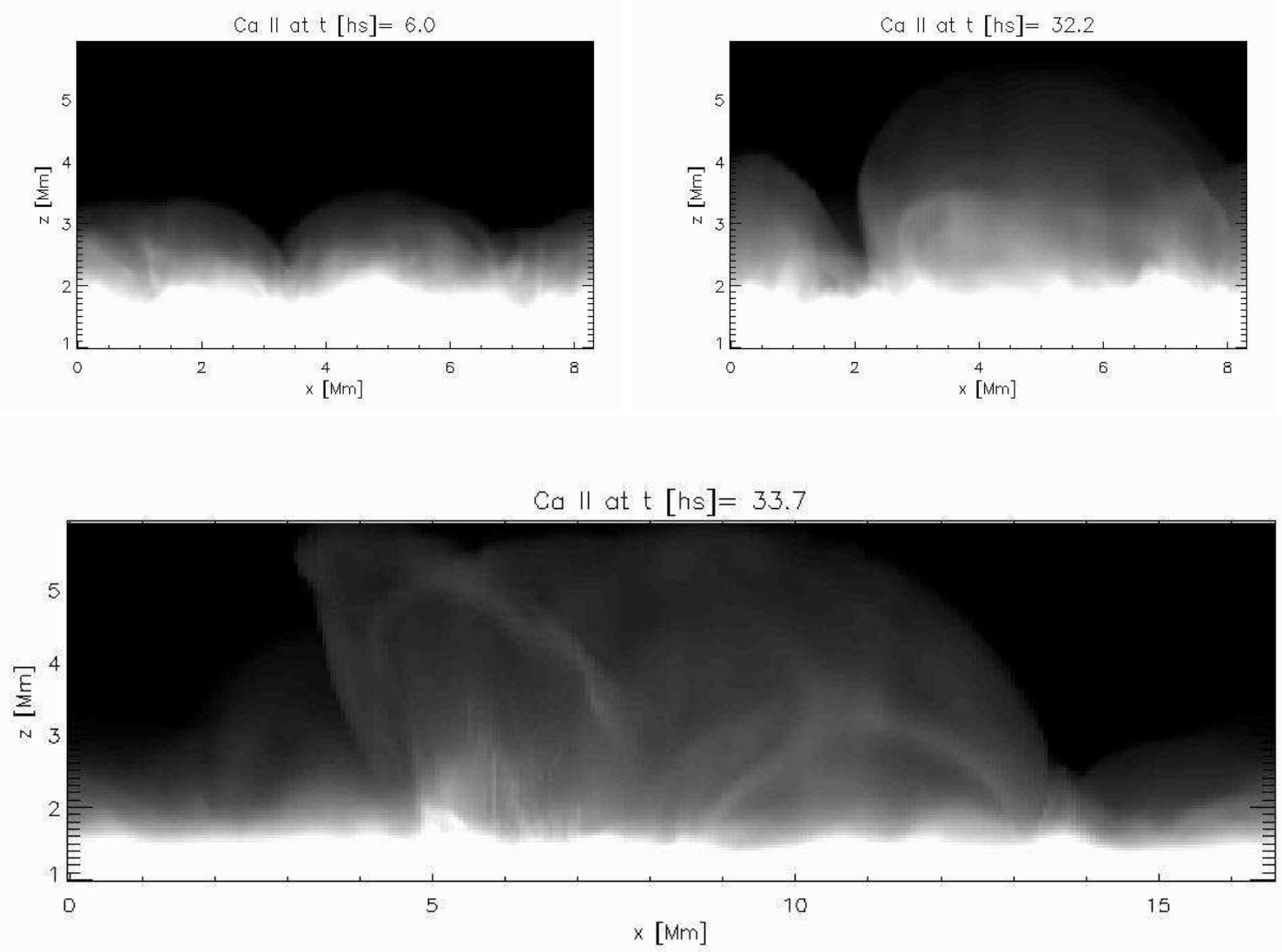

Fig. 20.- The synthetic Ca II H line Hinode intensity to the power of 0.25 (equivalent to a gamma of 0.25 in an image) is plotted to better bring out the structure of the bubble from simulation A4 at time $600 \mathrm{~s}$, before the flux tube goes through the chromosphere (top left), and at $3220 \mathrm{~s}$ when it has crossed the chromosphere (top right), and from the simulation B1 at time $3370 \mathrm{~s}$ (bottom) 

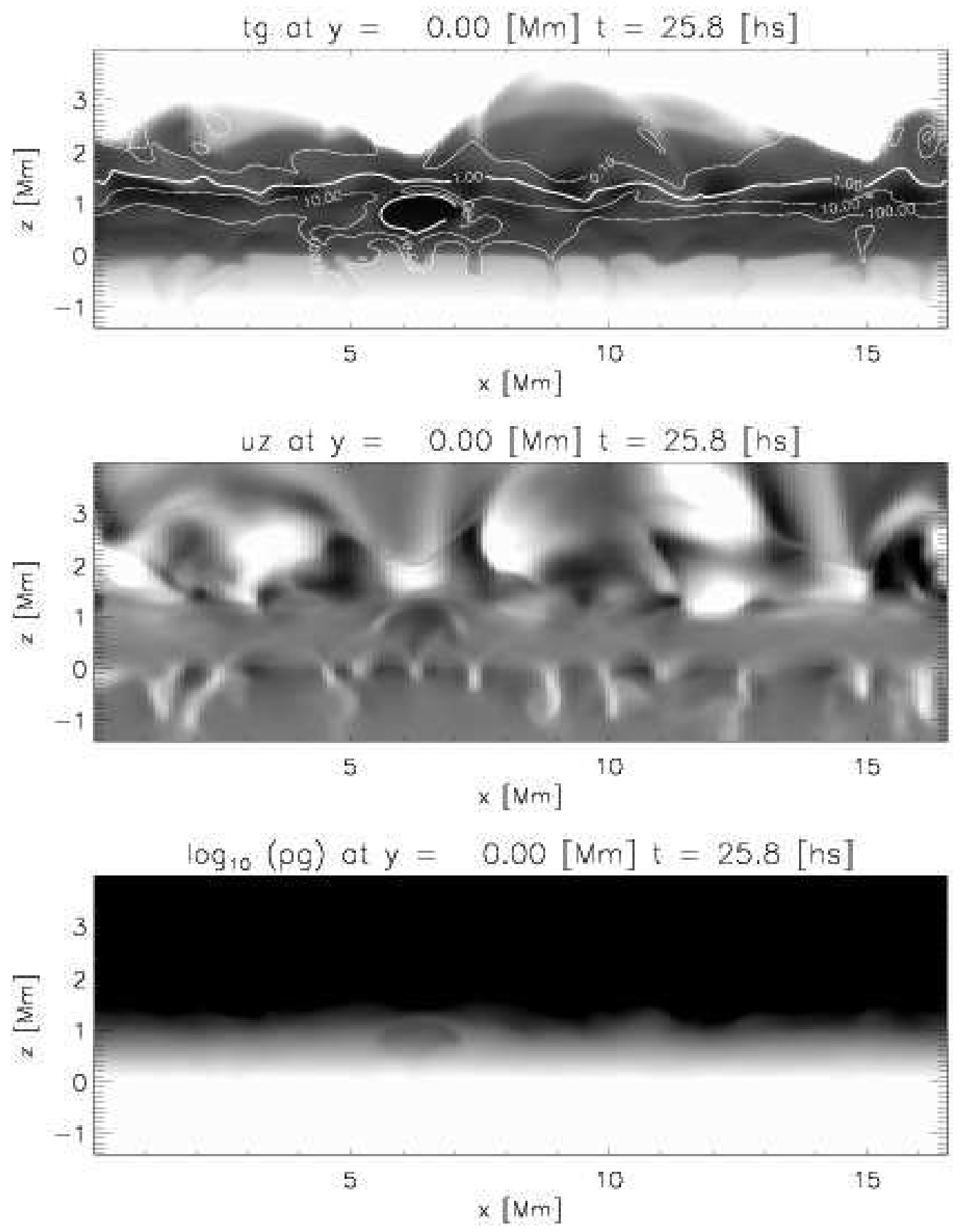

Fig. 21. - Vertical cuts of the logarithm of the temperature (top), vertical velocity (middle) and the logarithm of the gas pressure (bottom) from simulation B1 at time $t=2580 \mathrm{~s}$ and $y=0 \mathrm{Mm}$. Contours of plasma $\beta$ are overplotted the temperature. 

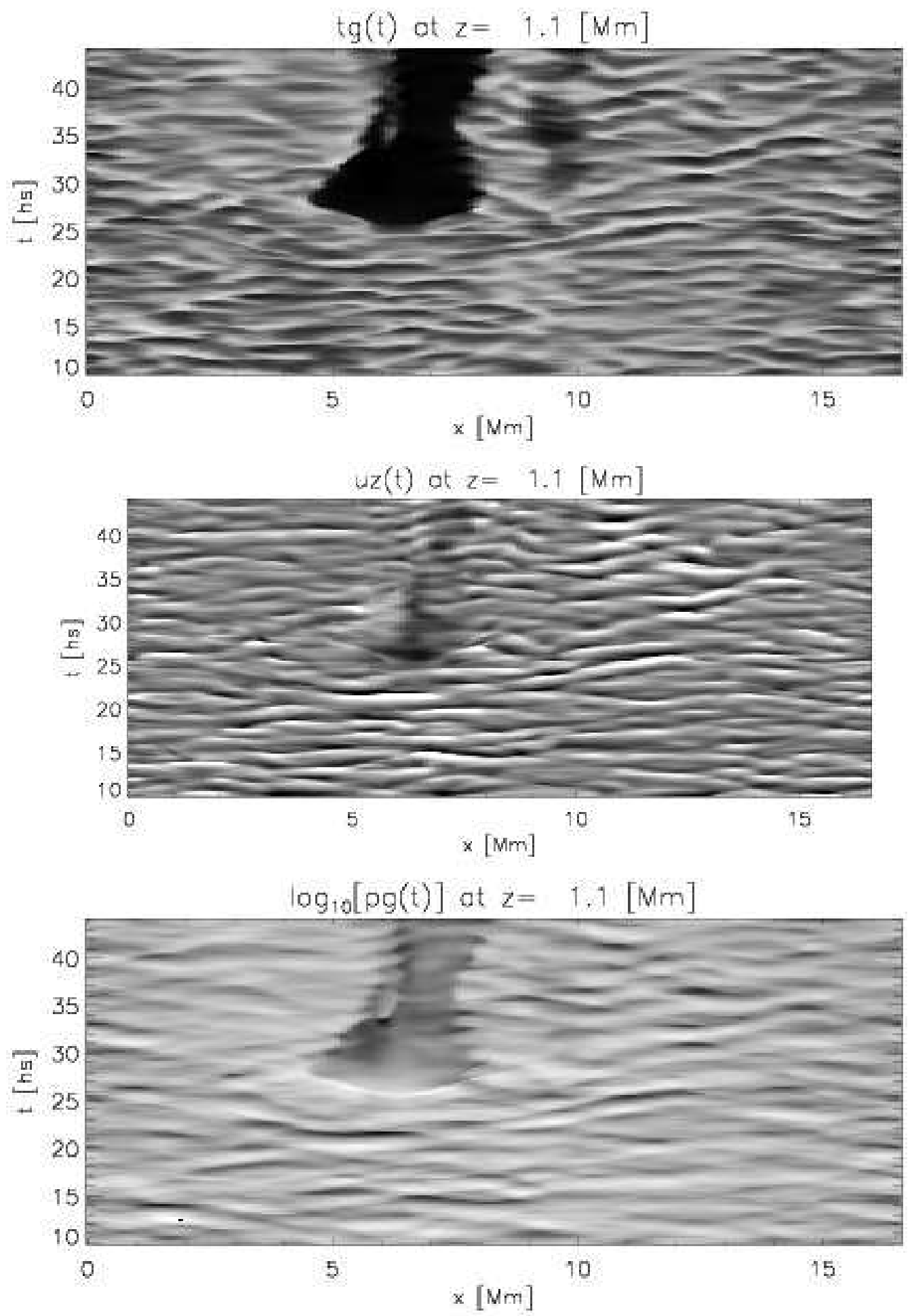

Fig. 22. - Temperature (top), vertical velocity (middle) and logarithm of the pressure (bottom) at a height of $1.1 \mathrm{Mm}$ as function of position in $x$ and time for a vertical cut at $y=0 \mathrm{Mm}$ for the simulation B1. 
Table 1. Simulation description

\begin{tabular}{cccccc}
\hline \hline Name & Twist $\lambda$ & $\mathrm{B}_{0}[\mathrm{G}]$ & Size & Comment & Time $[\mathrm{s}]$ \\
\hline A1 & 0.1 & 4484 & $8 \times 4 \times 16 \mathrm{Mm}^{3}$ & Flux tube in $y$ direction & 2200 \\
A2 & 0.2 & 3363 & $8 \times 4 \times 16 \mathrm{Mm}^{3}$ & Flux tube in $y$ direction & 2100 \\
A3 & 0.3 & 4484 & $8 \times 4 \times 16 \mathrm{Mm}^{3}$ & Flux tube in $y$ direction & 2500 \\
A4 & 0.6 & 4484 & $8 \times 4 \times 16 \mathrm{Mm}^{3}$ & Flux tube in $y$ direction & 3200 \\
B1 & 0.0 & 1121 & $16 \times 8 \times 16 \mathrm{Mm}^{3}$ & Flux sheet in $y$ direction. & 4500 \\
\hline
\end{tabular}


Table 2. time evolution

\begin{tabular}{lc}
\hline \hline \multicolumn{1}{c}{ Process } & Time $[\mathrm{s}]$ \\
\hline Expansion of granular cells in the photosphere & 900 \\
Expansion of the reverse granulation & 920 \\
Cooling of the center of granular cells in the photosphere & 1160 \\
Tube crosses the photosphere & 1300 \\
Tube crosses the chromospheric height forming reverse granulation & 1380 \\
Magnetic field moved to the intergranular lanes in the photosphere & 1550 \\
Reverse granulation cooling with expansion & 1560 \\
Tube crosses the layer $z=450$ km & 1660 \\
The cells return to "normal" size in the photosphere & 2100 \\
Upper chromosphere vertical expansion and cooling & 2900 \\
\hline
\end{tabular}

